\title{
Title:
}

\section{Intellectual disability-associated disruption of O-GIcNAcylation impairs neuronal development and cognitive function in Drosophila}

\section{Short title:}

\section{O-GIcNAc cycling controls cognitive function in Drosophila}

\author{
Michaela Fenckova ${ }^{1,2,5 \# *}$, Villo Muha ${ }^{1 \#}$, Daniel Mariappa ${ }^{1,3 \#}$, Marica Catinozzi ${ }^{4}$, Ignacy Czajewski ${ }^{1}$, \\ Laura E.R. Blok ${ }^{2}$, Andrew T. Ferenbach ${ }^{1}$, Erik Storkebaum ${ }^{4}$, Annette Schenck ${ }^{2 \S}$ and Daan M.F. \\ van Aalten $1 \S^{*}$ \\ 1Division of Gene Regulation and Expression, School of Life Sciences, University of Dundee, \\ Dundee, DD14HN, UK \\ 2Department of Human Genetics, Donders institute for Brain, Cognition and Behaviour, Radboud \\ University Medical Center, Nijmegen, 6525GA, Netherlands \\ ${ }^{3}$ Current address: Drosophila Genomics Resource Center, Biology Department, Indiana \\ University, Bloomington, Indiana 47405-7005, USA \\ ${ }^{4}$ Department of Molecular Neurobiology, Donders Institute for Brain, Cognition and Behaviour, \\ Faculty of Science, Radboud University, Nijmegen, 6525XZ, Netherlands \\ ${ }^{5}$ Current address: Department of Molecular Biology and Genetics, University of South Bohemia, \\ Ceske Budejovice, 37005, Czechia \\ \#these authors contributed equally \\ $\S$ shared last authors

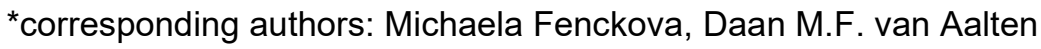 \\ Email: fenckm00@prf.jcu.cz \\ d.m.f.vanaalten@dundee.ac.uk
}

Keywords: O-GIcNAcylation/Drosophila/Intellectual disability/habituation/synapse 


\begin{abstract}
O-GIcNAcylation is a reversible co-/post-translational modification involved in a multitude of cellular processes. The addition and removal of O-GIcNAc modification is controlled by two conserved enzymes, O-GlcNAc transferase (OGT) and O-GIcNAc hydrolase (OGA). Mutations in OGT have recently been discovered to cause a novel Congenital Disorder of Glycosylation (OGT-CDG) that is characterized by intellectual disability. The mechanisms by which OGT-CDG mutations affect cognition remain unclear. We manipulated O-GIcNAc transferase and O-GIcNAc hydrolase activity in Drosophila and demonstrate an important role of O-GIcNAcylation in habituation learning and synaptic development at the larval neuromuscular junction. Introduction of patient-specific missense mutations into Drosophila O-GIcNAc transferase using CRISPR/Cas9 gene editing, leads to deficits in locomotor function and habituation learning. The habituation deficit can be corrected by blocking O-GIcNAc hydrolysis, indicating that OGT-CDG mutations affect cognitive function via reduced protein O-GIcNAcylation. This study establishes a critical role for O-GlcNAc cycling and disrupted O-GIcNAc transferase activity in cognitive dysfunction. These findings suggest that blocking O-GIcNAc hydrolysis is a potential treatment strategy for OGT-CDG.
\end{abstract}

\title{
Author summary
}

Attachment of single $\mathrm{N}$-acetylglucosamine (GlcNAc) sugars to intracellular proteins has recently been linked to neurodevelopment and cognition. This link has been strengthened by discovery of O-GlcNAc transferase (OGT) missense mutations in intellectual disability. Most of these mutations lie outside the catalytic O-GIcNAc transferase domain and it is unclear how they affect cognitive function. Using the fruit fly Drosophila melanogaster as a model organism, we found that a balance in O-GIcNAc cycling is required for learning and neuronal development. Habituation, a fundamental form of learning, is affected in flies that carry patient-specific OGT mutations, and increasing OGlcNAcylation genetically corrects the habituation deficit. Our work establishes a critical role for OGlcNAc cycling in a cognition-relevant process, identifies defective O-GIcNAc transferase activity as a cause of intellectual disability, and proposes underlying mechanisms that can be further explored as treatment targets.

\section{Introduction}

O-GlcNAcylation is an essential and dynamic co-/posttranslational modification that is characterized by attachment of $\mathrm{N}$-acetylglucosamine (GlcNAc) molecule to serine or threonine residues of intracellular proteins. O-GIcNAcylation is implicated in a wide range of cellular processes, such as: chromatin remodeling [1-3], transcription [4,5] and translation [6], Ras-MAPK and insulin signaling [7-9], glucose homeostasis [10], mitochondrial trafficking [11], and control of the circadian clock [12]. The addition and removal of O-GIcNAc modification, termed O-GIcNAc cycling, is controlled by two evolutionarily conserved enzymes, O-GIcNAc transferase (OGT) and O-GIcNAc hydrolase (OGA).

OGT, responsible for the addition of O-GIcNAc, is abundantly expressed in neurons and is enriched in the postsynaptic density (PSD) [13], a protein-dense structure that organizes the postsynaptic signaling machinery. O-GlcNAcylation is altered in brains of patients with Alzheimer's disease [14] and animal and cellular models of major neurodegenerative diseases [15-19]. In C. elegans models of neurodegeneration, O-GlcNAcylation protects against neurotoxicity [20]. Furthermore, it plays an important role in neuronal regeneration through the synchronization of insulin signalingdependent regenerative processes [8]. Recent studies also point to important functions in neuronal development, such as neuronal differentiation [21,22], assembly and axonal transport of 
neurofilaments [23], and synapse maturation [24]. Missense mutations in human OGT gene, located on the $X$ chromosome, are associated with intellectual disability (ID) [25-31], a severe neurodevelopmental disorder that is characterized by impaired cognition. Patients with OGT mutations suffer from a wide array of clinical features, including intrauterine growth retardation, developmental delay, delayed or restricted language skills and severe learning difficulties. The syndrome has been termed OGT-associated Congenital Disorder of Glycosylation (OGT-CDG) [32]. These findings and animal studies suggest that O-GlcNAcylation plays an important function in cognitive processes, such as learning [33,34].

The OGT protein consists of an N-terminal tetratricopeptide (TPR) domain, which contributes to substrate recognition and binding [35] and a C-terminal catalytic domain that is responsible for glycosylation of target proteins. OGT-CDG mutations are found in both domains. Unlike the mutations in the catalytic domain, the mutations in the TPR domain were shown to not significantly affect global protein O-GlcNAc levels but they do affect the OGT-TPR domain substrate binding and glycosylation kinetics, as derived from in vitro assays and crystal structure analysis [26,36,37]. However, it is not known whether impaired glycosylation is the mechanism that leads to developmental and cognitive defects caused by the TPR domain mutations. This question is pertinent as the TPR domain has been shown to be essential for cellular functions other than glycosylation [38].

Drosophila as a model organism has contributed to understanding the disease pathogenesis of numerous (ID) syndromes. It offers a combination of well-established gene targeting approaches and a plethora of morphological and functional disease-relevant phenotypes. The Drosophila OGT orthologue is encoded by the Polycomb group gene super sex combs (sxc) [39]. It is highly similar to human OGT[40]. Complete loss of sxc results in severe homeotic transformations of adult body structures and pupal lethality[41]. This lethality can be restored by ubiquitous overexpression of human OGT, demonstrating functional conservation [39]. sxc has also been associated with neuronal function in circadian rhythm regulation [42]. Therefore, Drosophila is highly suited to investigate the disrupted mechanisms underlying OGT-CDG.

Here we genetically manipulated both O-GIcNAc transferase and O-GIcNAc hydrolase activity using established sxc and Oga mutants and investigated the effect of O-GlcNAc cycling on cognitive function and neuronal development. We turned to habituation, an evolutionary conserved form of non-associative learning that is characterized by response decrement towards a repeated, non-meaningful stimulus. Habituation serves as a filter mechanism that prevents information overload and allows the cognitive resources to focus on relevant matters. It represents a prerequisite for higher cognitive functions ([43-45]. Deficits in habituation have been reported in a number of neurodevelopmental disorders [46] and in more than a hundred Drosophila ID models $[47,48]$. Neuronal development was investigated with the assessment of synaptic morphology at the Drosophila neuromuscular junction, an established model synapse [49]. To independently validate our findings we targeted the endogenous sxc locus by CRISPR/Cas9 editing [50] and generated a strong hypomorph mutation in the O-GIcNAc transferase domain ( $s \times c^{H 596 F}$ ). With the same technique we introduced three ID-associated missense mutations found in the conserved TPR domain of OGT: R284P [28], A319T [25] and L254F [29] and generated equivalent $s \times C^{R 313 P}$, $s X c^{A 348 T}$ and $s X c^{L 283 F}$ alleles, respectively. We evaluated their effect on protein O-GlcNAcylation, developmental viability, adult lifespan and locomotor activity, and assessed their effect on cognitive function and neuronal development.

We find that appropriate O-GlcNAc cycling is required for habituation, a fundamental form of learning that is widely disrupted in Drosophila models of ID, and for synaptic development. We show that OGT missense mutations, implicated in ID, outside the catalytic O-GIcNAc transferase 
domain lead to deficits in habituation learning and that these deficits are caused by disrupted OGIcNAc transferase activity. We thus unambiguously demonstrate the role of O-GlcNAcylation in brain development and function.

\section{Results}

\section{Alteration of O-GIcNAc transferase activity leads to a deficit in habituation}

To investigate whether the catalytic activity of Drosophila OGT is required for cognitive function, we tested the effect of an Sxc mutation with diminished catalytic activity, sXc ${ }^{H 537 A}$, on habituation. The homozygous hypomorphic flies are viable and except mild wing vein and scutellar bristle phenotypes do not present with any morphological abnormalities [50]. In habituation, an initial strong response towards a repeated but harmless stimulus gradually wanes based on prior experience. To assess this phenotype we used light-off jump habituation, an established nonassociative learning assay that meets the strict habituation criteria, including spontaneous recovery and dishabituation with novel stimulus and excluding sensory adaptation and motor fatigue[44,51]. We subjected $s \times c^{H 537 A}$ homozygous ( $s \times c^{H 537 A / H 537 A}$ ), heterozygous $\left(s \times C^{H 537 A /+}\right.$ ), and genetic background control flies (+/+) to 100 light-off pulses in the light-off jump habituation assay. While $s \times C^{H 537 A /+}$ and control flies exhibited good initial jump responses to the light-off stimuli $(61 \%$ and $67 \%$ initial jumpers out of $\mathrm{N}=96$ tested flies per genotype; above a required threshold of $50 \%$ [48]), $s \times c^{H 537 A / H 537 A}$ flies were impaired $(36 \%$ initial jumpers, $N=64)$, identifying broader defects that preclude assessment of habituation. Compared to control flies that habituated quickly to the repeated light-off stimulus, $s \times c^{H 537 A /+}$ flies displayed significantly slower habituation and failed to fully adapt their jump response during the entire course of the experiment (Figure 1A). These results suggest that partial loss of O-GIcNAc transferase activity or altered O-GIcNAcylation kinetics in $s \times C^{\mathrm{H} 537 \mathrm{~A} /+}$ mutants impairs habituation.

We validated our conclusion by employing a knockout-out allele of Drosophila Oga [52]. We asked whether partial inhibition of O-GlcNAc hydrolysis, by removing one copy of Oga $\left(\mathrm{Oga}^{\mathrm{KO} /+}\right)$ could improve habituation of the $s \times \mathrm{C}^{\mathrm{H} 537 \mathrm{~A} / \mathrm{+}}$ flies. Habituation of $\mathrm{Oga}^{\mathrm{KO} /+}$ flies was also slower but not significantly different from the control flies. The transheterozygous $s \times c^{\mathrm{H} 537 \mathrm{~A} /+} ; \mathrm{Oga} \mathrm{KO}^{\mathrm{O}+}$ flies showed good initial jump response, and their habituation was similar to control flies, identifying a significant improvement compared to $\mathrm{Sxc}^{\mathrm{H} 537 \mathrm{~A} / \mathrm{+}}$ flies (Figure 1B). The fatigue assay (see Materials and Methods) confirmed that the lower trials to criterion (TTC) values were not a result of increased fatigue (Figure S2A). These results show that Drosophila is a suitable model to study the role of OGT in cognitive functioning and demonstrate that tight control of protein O-GlcNAcylation is required for proper habituation learning in Drosophila.

\section{O-GIcNAc transferase is required for habituation in neurons}

We next sought to determine whether the $S x \mathrm{C}^{\mathrm{H} 537 \mathrm{~A} /+}$ habituation deficit originates from reduced OGT function in neurons. We therefore induced neuronal knockdown of $s x c$ by crossing the pan-neuronal elav-Gal4 driver line (see Material and Methods) to an inducible RNAi line against sxc obtained from Vienna Drosophila Resource Center (\#18610, zero predicted off-targets). Progeny from crossing the driver line to the isogenic genetic background of the RNAi line (\#60000) were used as controls. While control flies (elav-Gal4/+) showed good initial jump response $(56 \%, N=64$ ), elavGal4>UAS-sXC $C^{R N A i}$ flies - similar to $s \times c^{H 537 A / H 537 A}$ flies - exhibited very low initial jump response to light-off stimulus ( $19 \%$ initial jumpers, $N=96$ ). While this detrimental effect prevented assessment of $s x c$ neuron-specific knockdown in habituation learning, it does argue that i) the failed jump 
response of $s \times c^{H 537 A / H 537 A}$ flies is likely due to loss of OGT activity in neurons, and ii) OGT activity is indispensable for basic neuronal function or neuronal development.

We used an alternative strategy to test whether habituation deficits of $s \times c^{\mathrm{H} 537 \mathrm{~A} / \mathrm{f}}$ flies are of neuronal origin. We asked whether restoration of OGT activity in neurons can correct habituation deficits of sxc ${ }^{H 537 A /+}$ flies, by inducing pan-neuronal overexpression of functional wild-type sxc in the heterozygous $s x c^{H 537 A /+}$ as well as control background. Neuronal overexpression of functional sxc in control flies (elav-Gal4>UAS-sxc) resulted in a habituation deficit (Figure 1C). This is consistent with our previous findings of habituation deficits in Oga $\mathrm{KO} / \mathrm{KO}$ flies, which also show increased protein O-GIcNAcylation [52]. In contrast, re-expression of functional $s x c$ in the $s x c^{H 537 A /+}$ flies completely corrected their habituation deficits (Figure 1D). The lower TTCs were not a result of increased fatigue (Figure S2B). Therefore, appropriate levels of OGT activity and O-GIcNAcylation, specifically in neurons, are required for habituation learning.

\section{Neuronal O-GIcNAc transferase activity controls synaptic development}

Synapse biology is important for brain development and cognition, and abnormalities in synaptic architecture are characteristic of multiple Drosophila models of neurodevelopmental disorders [5358]. For these reasons, we asked whether $s \times \mathrm{C}^{\mathrm{H} 537 \mathrm{~A} / \mathrm{+}}$ mutants show any defects in the morphology of the third instar larval neuromuscular junction (NMJ), a well-established model synapse. We labeled NMJs by immunostaining for the postsynaptic membrane marker anti-discs large (Dlg1) to visualize the overall morphology of the NMJ terminal, and for synaptotagmin (Syt), a synaptic vesicle marker that visualizes synaptic boutons. We did not observe a significant change in synaptic length, area, or perimeter (Figure 2A), nor in the number of branches and branching points (Figure S3A) in NMJs of $s \times C^{H 537 A /+}$ mutant larvae but we observed a significant increase in bouton number compared to the genetic background control (Figure 2A). Increasing OGT activity by presynaptic overexpression of wild-type sxc (elav-Gal4>UAS-sxc) resulted in an opposite phenotype: a decreased number of boutons compared to both controls, UAS transgene and driver alone. NMJ length was also reduced (Figure 2B). Both parameters were normalized when we neuronspecifically expressed functional $s \times c$ in the $s \times C^{H 537 A /+}$ mutant background ( $s \times C^{H 537 A /+}$, elavGal4>UAS-sxc) (Figure 2C). These results demonstrate a role of $s x c$ in the synaptic bouton number and NMJ morphology and indicate that tight control of O-GlcNAcylation is important for proper synaptic development.

The decrease of synaptic length caused by neuronal overexpression of $s x c$ in the control but not $s \times c^{H 537 A /+}$ background indicates that strong dysregulation of $s \times c$ might be required to uncover its function in synaptic growth. Accordingly, when we investigated homozygous sxc ${ }^{H 537 A}$ larvae, we found a significant increase in NMJ length and perimeter. However, the increase in the number of synaptic boutons did not reach significance (Figure S3D). Therefore, to validate the synaptic bouton and length/growth phenotypes, we used CRISPR/Cas9 gene editing to generate a stronger catalytic hypomorph, $s \times C^{H 596 F}$ (Figure S1A,B). The in vitro catalytic activity of $s \times C^{H 596 F}$ was reported to be $3 \%$ relative of normal OGT activity, less than the reported catalytic activity of $s \times C^{H 537 A}(5.6 \%$ activity) [40]. Indeed, we found total O-GIcNAc levels in sxc ${ }^{H 596 F}$ homozygous embryos to be reduced (Figure S4A). sXC ${ }^{H 596 F}$ homozygous flies are viable, confirming minimal requirement of endogenous OGT activity for completion of development in Drosophila (Figure S4B). We found that NMJs of sXC ${ }^{H 596 F}$ larvae display a significant increase in synaptic bouton number, NMJ length, area and perimeter (Figure 2D), reflecting a more severe NMJ phenotype. We also subjected the $s \times c^{H 596 F}$ flies to light-off jump habituation assay but homozygous as well as heterozygous $s \times c^{H 596 F}$ 
flies showed impaired jump response ( $38 \%$ and $42 \%$ initial jumpers), similar to homozygous sXc ${ }^{H 537 A}$ and pan-neuronal sxc knockdown flies. This precluded the assessment of habituation.

A knockout of Oga normalized the bouton numbers and partially also the area and perimeter of the sxc H596F NMJs (Figure 2D). These data show that O-GIcNAcylation controls bouton number and partially also NMJ size.

\section{Characterization of development and locomotor function of sxc mutations associated with Intellectual Disability}

Recent studies have reported three hemizygous missense mutations (R248P, A319T, L254F) in human OGT in male individuals with ID. The de novo R248P mutation was identified by trio whole exome sequencing in an affected individual with ID and developmental delay [28]. A319T and L254F mutations were identified by $X$ chromosome exome sequencing. The A319T mutation, present in three individuals with severe ID, was inherited from the mother but segregated with an uncharacterized missense mutation in MED12, a gene already implicated in ID [25]. L254F was present in three related individuals with moderate to mild ID $[27,29]$. These mutations reside in the conserved TPR domain, outside of the catalytic O-GIcNAc transferase domain [25,27-29]. In order to investigate the functional consequences of these mutations, we introduced the equivalent missense mutations (R313P, A348T, L283F) into the sxc gene using CRISPR/Cas9 editing (Figure S1) and generated three novel $s X C$ ID alleles $s X C^{R 313 P}$, $s X C^{A 348 T}$, and $s X c^{L 283 F}$.

We first characterized the development of the patient-related mutant $s \times c$ alleles. We transferred embryos at stage 11-16 to vials with fresh food and counted the number of resulting pupae and adult flies. Homozygous $s \times c^{R 313 P}, s \times c^{A 348 T}$, and $s \times c^{L 283 F}$ embryos developed normally to adulthood without apparent delay and the percentage of pupae and adults did not statistically differ from the genetic background controls (Figure 3A).

We next investigated locomotor phenotypes in adult $s x c^{R 313 P}, s x c^{A 348 T}$, and $s x c^{L 283 F}$ flies using the island and negative geotaxis assays. In the island assay, flies were thrown onto a white platform surrounded by water, and the number of individuals remaining on the platform was quantified over time. Homozygous $s \times c^{R 313 P}, s X c^{A 348 T}$ and $s \times c^{L 283 F}$ flies escaped from the platform with similar efficiency as the genetic background control (Figure 3B), indicating that their startle response is not affected.

In the negative geotaxis assay, climbing performance of homozygous and heterozygous $s \times c^{R 313 P}$ flies was significantly slower while homozygous $s \times c^{A 348 T}$ and $s X c^{L 283 F}$ flies showed an average climbing speed similar to the control (Figure 3C). We also tested Drosophila lines with homozygous H537A ( $s \times c^{H 537 A}$ ) or H596F ( ${ }^{\text {X }} \mathrm{C}^{\mathrm{H} 596 \mathrm{~F}}$ ) catalytic hypomorph mutations and they exhibited similar climbing speed as the control group (Figure 3C). This suggests that the R313P mutation affects coordinated locomotor behavior in the negative geotaxis assay by other mechanisms than $\mathrm{O}-$ GlcNAc transferase activity.

Taken together, similar to catalytic hypomorphs ([50] and Figure S2B) the $s \times C^{R 313 P}, s X C^{A 348 T}$, and $s X C^{L 283 F}$ mutants are fully viable and develop normally to adulthood. Neither the reduction of protein O-GIcNAcylation induced by catalytic hypomorph alleles nor the $s x c^{A 348 T}$ and $s x c^{L 283 F}$ ID alleles cause severe locomotor defects in adult flies. Only the $s \times C^{R 313 P}$ allele negatively affects climbing performance. This effect appears to be independent of O-GIcNAc transferase activity. However, a 
contribution of a potential second site mutation, not specific to sxc gene, that was not be eliminated by six generations of backcrossing cannot be excluded.

\section{Patient-related sxc mutant alleles do not affect global protein O-GIcNAcylation}

We investigated whether the ID-associated alleles in sxc affect protein O-GlcNAcylation, by subjecting lysates from adult heads of $s \times C^{R 313 P}$, $s \times c^{A 348 T}$, and $s \times c^{L 283 F}$ flies to Western blotting. Labeling with anti-O-GlcNAc antibody (RL2) that is able to capture O-GlcNAcylation changes in the catalytic hypomorphs (Figure S4B and [40]) indicated that the levels of protein O-GlcNAcylation are not significantly altered in each of the three mutants (Figure 3D,E). This is in line with normal levels of O-GlcNAcylation in patient-derived fibroblasts and human embryonic stem cell models of the R313P and L283F equivalent mutations [26,28]. O-GlcNAcylation of the human A348T equivalent has not been investigated. The cell models of OGT-CDG mutations show downregulation of Oga, which may compensate for decreased O-GlcNAcylation. Although existence of such regulatory mechanism has not been shown in Drosophila, we analyzed the OGlcNAcylation levels in Oga knockout background. Blocking O-GlcNAc hydrolysis in patientrelated sxc mutant alleles with Oga ${ }^{K O}$ increased O-GIcNAc levels to the same degree as in OgaKO samples (Figure 3D,E). We thus conclude that ID-associated OGT mutations do not have a grossly affected protein O-GIcNAcylation.

\section{$s \times c^{R 313 P}$ and $s \times c^{A 348 T}$ display defective habituation learning}

Because of their role in ID in humans, we also investigated the effect of the novel $s X C^{R 313 P}, s X C^{A 348 T}$, and $s \times c^{L 283 F}$ alleles on habituation learning. We first subjected the $s \times c^{L 283 F}$ flies to 100 light-off pulses in the habituation assay. Despite sufficient locomotor abilities to perform in the island test and negative geotaxis assays, the initial jump response of the $s x c^{L 283 F}$ homozygous and heterozygous flies was below the required threshold of $50 \%$ therefore deemed non-performers ( $s \times C^{L 283 F / L 283 F}$ : $36 \%$ initial jumpers, $N=96$; $s X C^{L 283 F /+}: 47 \%$ initial jumpers, $N=96$ ). Insufficient performance at the beginning of the assay thus precluded the assessment of habituation in these flies. We observed the same phenotype also for the $s \times c^{R 313 P}$ homozygous flies ( $49 \%$ initial jumpers, $N=64)$. The initial response in $s X C^{R 313 P}$ heterozygous flies was sufficient $(67 \%)$ and they were not able to suppress their jump response to the repeated light-off stimuli as efficiently as the genetic background control flies (Figure 4A), revealing a learning deficit. Flies heterozygous for the $s \times c^{A 348 T}$ allele showed a good initial jump response and habituated similar to the control, while $s x c^{A 348 T}$ homozygous flies showed a habituation deficit (Figure 4B). In summary, deficits in habituation learning were observed for the R313P (heterozygous) and A348T (homozygous) mutations, while evaluation of the L254F homo- and heterozygous as well as R313P homozygous conditions was precluded.

\section{Blocking O-GIcNAc hydrolysis corrects habituation deficits of $s \times c^{R 313 P}$ and $s \times c^{A 348 T}$}

The apparently unaltered levels of O-GlcNAcylation in ID-associated sxc mutants (Figure 3D,E) may suggest that O-GIcNAc-independent mechanisms underlie their cognitive phenotypes. To test this experimentally, we performed the habituation assay in flies carrying $s \times c^{R 313 P}$ allele and either the Oga ${ }^{K O}$ allele or an Oga mutation that specifically blocks its O-GlcNAc hydrolase activity $\left(\mathrm{Oga}^{D 133 N}\right)$. Notably, heterozygous $\mathrm{Oga}^{K O}$ and $\mathrm{Oga}^{D 133 N}$ flies habituated to a similar degree as the genetic background control flies and when combined with the heterozygous, habituation deficient $s \times c^{R 313 P}$ allele, fully rescued defects seen in the $s x c$ mutants alone (Figure 4C,D). Similarly, we attempted a rescue of habituation deficient homozygous $s \times c^{A 348 T}$ with homozygous Oga ${ }^{K O}$ and Oga ${ }^{D 133 N}$ alleles. We have previously shown that these also exhibit habituation deficits [52]. 
Strikingly, blocking O-GlcNAc hydrolysis by Oga ${ }^{K O / K O}$ or Oga ${ }^{D 133 N / D 133 N}$ in SxC $^{A 348 T / A 348 T}$ flies was sufficient to completely rescue habituation deficits of either single mutant condition (Figure 4E,F). All tested flies show a good initial jump response and lower TTCs in the rescue experiments were not caused by fatigue (Table S2, Figure S4). In summary, despite seemingly normal gross OGIcNAc levels in the $s \times C^{R 313 P}$ and $s \times c^{A 348 T}$ ID alleles, these genetic experiments provide evidence that their deficits in habituation learning depend on defective OGT enzymatic activities.

$s \times c^{R 313 P}$ and $s \times c^{A 348 T}$ and $s \times c^{L 283 F}$ show deficits in synaptic morphology

We subjected homozygous $s \times c^{R 313 P}$ and $s X c^{A 348 T}$ and $s \times C^{L 283 F}$ larvae to assessment of synaptic morphology at the NMJ. We found that the NMJs of all three patient-related sxc mutant alleles display significant increase in synaptic bouton numbers and $s \times C^{R 313 P}$ larvae also display significantly increased length and perimeter of the NMJ. The length is also increased in the $s \times c^{A 348 T}$ and $s X c^{L 283 F}$ larvae albeit not significant (Figure 5A). In addition, $s X c^{R 313 P}$ and $s x c^{L 283 F}$ larvae show increased number of synaptic branches (Figure S5). Overall, the NMJ morphology phenotypes of the patient-related $s \times c$ mutant alleles resemble those of the catalytic hypomorphs $\mathrm{sxC}^{\mathrm{H} 537 \mathrm{~A} / \mathrm{+}}$ (Figure 2A) and $s \times c^{H 596 F / H 596 F}$ (Figure 2D). This is in line with the observation that the patient-related sXC ${ }^{R 313 P}$ and $S X C^{A 348 T}$ alleles affect O-GIcNAc transferase activity, which is indispensable for habituation (Figure 4C-F). The shared NMJ phenotype signature between the catalytic and patientrelated mutants is the increase of synaptic bouton number. Because R313P and L283F mutations also significantly affect other NMJ parameters, we conclude that they are stronger/more detrimental than A348T mutation. This is in line with the observed effect on the jump performance in the lightoff jump habituation assay ( $s X C^{L 283 F / L 283 F}, s X c^{283 F /+}$ and $s X C^{R 313 P / R 313 P}$ non-performers).

The Drosophila O-GIcNAc proteome is enriched in genes with function in neuronal development, learning \& memory, and human ID gene orthologs

ID-associated mutations in sxc do not globally reduce protein O-GIcNAcylation yet blocking OGIcNAc hydrolysis can completely restore the learning deficits in light-off jump habituation. We hypothesized that altered O-GlcNAcylation of specific sxc substrates is responsible for the habituation deficits. We therefore attempted to predict candidate substrates by exploration of the Drosophila O-GIcNAc proteome, as previously determined through enrichment with a catalytically inactive bacterial O-GIcNAcase [59]. We first performed an enrichment analysis of neuronal and cognitive phenotypes (as annotated in Flybase, see Materials and Methods) among the Drosophila O-GIcNAc substrates (encoded by in total 2293 genes) and found that they are significantly enriched in phenotype categories learning defective (Enrichment $=1.5, p_{\text {adj }}=0.032$ ), memory defective $\left(\right.$ Enrichment $\left.=1.5, p_{\text {adj }}=0.016\right)$, neurophysiology defective $\left(\right.$ Enrichment, 1.5, $p_{\text {adj }}=$ $3.2 \times 10^{-5}$ ) and neuroanatomy defective (Enrichment $=1.9, p=5.27 \times 10^{-35}$ ) (genes listed in Table S3), supporting the importance of O-GIcNAcylation for neuronal development and cognitive function. We also found that human orthologs of 269 genes from the O-GIcNAc proteome are proven or candidate monogenic causes of ID (Enrichment $\left.=1.7, p_{\text {adj }}=1.01 \times 10^{-16}\right)$. When restricting this analysis to proteins with high-confidence mapped O-GIcNAc sites (in total 43) [59], we found orthologs of nine O-GlcNAcylated proteins to be implicated in Intellectual Disability, again representing a significant enrichment (Enrichment $\left.=3.4, p_{\text {adj }}=0.01\right)$. These orthologs are: Atpalpha (human ATP1A2), Gug (human ATN1), Hcf (human HCF1), LanA (human LAMA2), mop (human PTPN23), NAChRalpha6 (human CHRNA7), Ndg (human NID1), Nup62 (human NUP62) and Sas4 (human CENPJ). They represent potential downstream effectors of sxc that may control habituation learning in wild-type condition and may contribute to habituation deficits in catalytic and ID-associated sxc mutant conditions. Further analysis will be required to answer the question 
whether impaired O-GICNAc activity towards one or more of these targets is responsible for habituation deficits that are associated with OGT-CDG mutations.

\section{Discussion}

O-GIcNAcylation is important for habituation and for neuronal development in Drosophila Habituation, the brain's response to repetition, is a core element of higher cognitive functions $[43,44,60]$. Filtering out irrelevant familiar stimuli as a result of habituation allows to focus the cognitive resources on relevant sensory input. Abnormal habituation was observed in a number of neurodevelopmental disorders, including ID and Autism [46] and characterizes $>100$ Drosophila models of ID $[47,48]$. To address the role of OGT and its O-GIcNAc transferase activity in this cognition-relevant process, we investigated heterozygous $s x c^{H 537 A /+}$ flies [50] in light-off jump habituation. We found that they were not able to suppress their escape behavior as a result of deficient habituation learning (Figure 1A). This finding is in line with the recently published habituation deficit of the complete knock-out of OGT ortholog in C. elegans [61] and shows that altering O-GIcNAc transferase activity is sufficient to induce this deficit. We thus demonstrate the importance of O-GIcNAcylation in habituation learning.

Proper development and maintenance of synapses is an important aspect of neuronal function and cognition. The synaptic connection between motor neurons and muscle cells, termed the neuromuscular junction (NMJ), represents an excellent model system to study the molecular mechanisms of synaptic development in Drosophila [62]. Because NMJ defects were found in several Drosophila disease models with defective habituation [47,53-55], we investigated the synaptic architecture of the $s \times c^{H 537 A /+}$ larvae. We found that NMJs of the $s \times c^{H 537 A /+}$ larvae are characterized by an increased number of synaptic boutons, recognizable structures that contain the synaptic vesicles (Figure 2A). Larvae with a stronger homozygous catalytic mutation, sXC $C^{H 596 F / H 596 F}$, also show an increase in NMJ length, area, and perimeter. We conclude that OGlcNAcylation is important for control of synaptic size and synaptic bouton number.

\section{Appropriate O-GIcNAc cycling is required for habituation learning and maintenance of the synaptic size}

We recently showed that increased protein O-GlcNAcylation in homozygous Oga knockout flies causes a habituation deficit [52]. Here we show that heterozygous Oga knockout can restore the habituation deficit of $s \times c^{\mathrm{H} 537 \mathrm{~A} / \mathrm{+}}$ flies (Figure 1B). This indicates that habituation learning depends on O-GlcNAc cycling. Because the loss of one Oga allele does not significantly affect total OGIcNAc levels [52], we presume that subtle changes in O-GlcNAcylation dynamics rather than gross loss of O-GIcNAc transferase activity inhibits habituation learning.

It is known that postsynaptic expression of OGT in excitatory synapses is important for synapse maturity in mammals [24]. Here we show that presynaptic O-GlcNAc transferase also has role in synapse growth. At the NMJ, the synapses of larvae with neuronal overexpression of sxc are shorter, and the number of synaptic boutons is decreased (Figure 2B). Both length and bouton number are normalized when sxc is overexpressed in neurons of the sxc ${ }^{H 537 A /+}$ larvae (Figure 2C). This phenotype was not observed in Oga knockout larvae with increased O-GlcNAcylation. Knockout of Oga can correct the increased bouton number in larvae with sxc ${ }^{\text {H596F }}$ mutation but not the NMJ size (Figure 2D).

Our data suggest that the NMJ defects associated with decreased O-GlcNAc transferase function are of neuronal origin and that O-GIcNAcylation controls the number of synaptic boutons and partially also synaptic size. Absence of synaptic size defects in Oga knockout larvae and failure of 
Oga ${ }^{K O}$ to rescue the NMJ size defects caused by decreased O-GlcNAcylation indicates that other, non-catalytic O-GIcNAc transferase functions may be involved in control of synaptic size. Levine et al. recently demonstrated that non-catalytic activities of OGT are necessary for its function in some cellular processes, such as proliferation [38].

\section{Drosophila NMJ as a model for the O-GIcNAc-related synaptopathy}

The $s \times c$ catalytic hypomorph mutations ( $s \times c^{\mathrm{H} 537 \mathrm{~A}}$, $\left.s \times c^{\mathrm{H} 596 \mathrm{~F}}\right)$ as well as the OGT-CDG-patient equivalent mutations (R284P, A319T, L254F) that we introduced with the CRISPR/Cas-9 geneediting technology in the Drosophila $s \times c$ gene ( $\left.s \times c^{\mathrm{R} 313 \mathrm{P}}, s \times c^{\mathrm{A} 348 \mathrm{~T}}, s \times c^{\mathrm{L} 283 F}\right)$, lead to an increase in the number of synaptic boutons, and in some cases also to an increase in synaptic size. NMJ size and the number of synaptic boutons in our model is determined by the level of sxc activity. Dependence of these parameters on gene activity/dosage was previously established for Fmr1 (Drosophila model of Fragile X Syndrome) and other genes. Fmr1 and VAP-33A (implicated in Amyotrophic Lateral Sclerosis) control synaptic growth and bouton expansion through presynaptic organization of microtubule architecture [58,63]. In gain and loss-of-function models of neurodevelopmental disorders caused by SHANK3 mutations, postsynaptic regulation of Wnt signaling has been identified as an underlying mechanism [64]. Lastly, canonical and non-canonical BMP signaling is responsible for the observed bouton number deficits associated with Neuroligin4 (implicated in ID and ASD) and highwire (ubiquitin ligase) [65,66]. These mechanisms are tightly linked to synaptic activity, and might also dynamically react to changes in synaptic strength [6769]. It will be interesting to further investigate whether these mechanisms are affected by catalytic and patient-related sxc mutations and by Oga mutations. In particular, the potential involvement of microtubules deserves attention. It has been shown that O-GIcNAcylation of tubulin negatively regulates microtubule polymerization and neurite outgrowth in mammalian cell lines [70]. Impaired Drosophila OGT catalytic activity may thus cause the synaptic length phenotypes by increased microtubule polymerization.

Increased number of synaptic boutons has also been associated with increased excitability at the NMJ [71]. Future studies could employ electrophysiological assessment of NMJ activity and explore whether the O-GIcNAc cycling and patient-related sxc mutations affect neuronal excitability.

\section{Mutations implicated in OGT-CDG affect habituation via modulation of O-GICNAC transferase activity}

We assessed the effect of OGT-CDG missense mutations on habituation. We found that $s \times c^{\mathrm{R} 313 P}$ and $s \times c^{\mathrm{A} 348 \mathrm{~T}}$ inhibit habituation in the light-off jump habituation assay (Figure $4 \mathrm{~A}, \mathrm{~B}$ ). $s \times c^{\mathrm{L} 283 \mathrm{~F}}$ could not be investigated as these mutants displayed a non-performer phenotype in the light-off jump response. While the full spectrum of ID-related phenotypes in an individual with R284P mutation has been attributed to OGT, the A319T mutation segregates with an uncharacterized missense mutation in another gene implicated in ID, MED12 (G1974H) [25]. It was elusive which of the mutations is responsible for ID in the affected individuals. We provide evidence that the Drosophila equivalent of the A319T mutation in the TPR domain of OGT causes a cognitive deficit and support a causal role of A319T in OGT-CDG.

Consistent with no detectable O-GIcNAc changes in patient samples and cellular models of the non-catalytic OGT mutations $[26,28]$, no appreciable reduction in protein O-GlcNAcylation was observed in $s \times c^{R 313 P}$ and $s \times c^{A 348 T}$ flies. However, habituation learning was restored by increasing O-GlcNAcylation through blocking Oga activity (Figure 4C-F). This argues that the mechanism by which $s \times c^{\mathrm{R} 313 \mathrm{P}}$ and $s \times c^{\mathrm{A} 348 \mathrm{~T}}$ inhibit habituation is defective O-GIcNAc transferase activity, paralleling impaired O-GIcNAc transferase activity and significant reduction of protein O-GlcNAcylation 
demonstrated in the catalytic OGT-CDG mutations [30,31]. It is worth noticing that we have previously shown that mutations in Oga also cause habituation deficits [52]. Our finding that genetic combination of loss of OGA with loss of OGT activity rescues the cognitive readout argues that OGA inhibition using available inhibitors may represent a viable treatment strategy. The R284P and A319T reside in the TPR domain (Figure S1) which is responsible for recognition and binding of OGT substrates [38,72,73]. All OGT-CDG mutations investigated in this study were shown to impair the substrate interaction properties and the glycosyltransferase kinetics [27,36]. The observed habituation deficits may thus be caused by impaired O-GIcNAcylation dynamics towards a specific set of substrates that cannot be captured by standard O-GlcNAc detection assays. Identification of these substrates may pinpoint the underlying defective mechanisms and additional treatment targets.

Our explorative analysis found that of 43 O-GIcNAcylated proteins [59], nine are orthologs of human proteins implicated in ID: ATP1A2, ATN1, HCF1, LAMA2, PTPN23, CHRNA7, NID1, NUP62 and CENPJ. They represent potential downstream effectors and can be investigated in future studies. Particularly the transcriptional co-regulator HCF1 (Host Cell Factor 1) emerges as a top candidate. In mammals, OGT mediates glycosylation and subsequent cleavage of HCF1, which is essential for its maturation [74]. Recombinant OGT with R284P mutation is defective in HCF1 glycosylation [28] and HCF1 processing was shown to be completely abrogated by a catalytic OGTCDG mutation [30]. Drosophila sxc is also a known regulator of polycomb gene function. Many polycomb regulatory genes have been implicated in ID [42]. For example, Gug (human ATN1), a polycomb co-regulator [75] from the embryonic O-GIcNAc proteome may link cognitive deficits of OGT-CDG mutations to polycomb gene function.

In summary, we show that OGT-CDG mutations in the TPR domain negatively affect habituation learning in Drosophila via reduced protein O-GIcNAcylation. The data support a causal role of A319T in OGT-CDG and demonstrate that Drosophila habituation can be used to analyze the contribution of OGT mutations to cognitive deficits. This important aspect of ID has to date not been addressed for any of the OGT-CDG mutations. Moreover, our genetic approach points to a key role of O-GIcNAc transferase activity in ID-associated cognitive deficits and identifies blocking OGIcNAc hydrolysis as a treatment strategy that can ameliorate cognitive deficits in OGT-CDG patients. Thanks to its high-throughput compatibility, the light-off jump habituation assay can be used with high efficiency for future identification of the downstream effectors and novel therapeutic targets for OGT-CDG.

\section{Materials and Methods}

\section{Cloning of the guide RNA and repair template DNA vectors for Drosophila CRISPR/Cas9 editing}

Novel mutant Drosophila lines, $s \times C^{H 596 F}, s x c^{R 313 P}, s x c^{A 348 T}$, and $s X C^{L 283 F}$, were generated via CRISPR/Cas9 gene editing, following a previously described protocol [50]. Briefly, guide RNA sites were selected using an online tool (crispr.mit.edu) and the annealing primer pairs with appropriate overhangs for Bpil restriction digestion were cloned into pCFD3-dU63gRNA plasmid [76]. Vectors coding for repair template DNA of roughly $2 \mathrm{~kb}$ were generated from Drosophila Schneider 2 cell genomic DNA by PCR using GoTaq G2 Polymerase (Promega) and primer pairs appropriate for the desired region (Supplementary Table S1). The PCR products were digested with Bpil and inserted into the PGEX6P1 plasmid. The intended mutation, as well as silent mutations required to remove the gRNA sequence (Figure S1), were incorporated by either site-directed mutagenesis 
(H596F) using the QuikChange kit (Stratagene) or restriction-free cloning (R313P, A348T and L283F) [77]. The four sets of mutations - H596F, L283F, R313P, and A348T removed restriction sites for Hinfl, Bfml, Mnll and Bsql, respectively. DNA products of cloning and mutagenesis were confirmed by sequencing. All primer sequences are listed in Supplementary Table S1.

\section{Generation of $s \times c^{H 596 F}, s \times c^{R 313 P}, s \times c^{A 348 T}$, and $s \times c^{L 283 F}$ Drosophila lines}

Vasa::Cas9 Drosophila embryos (strain \#51323 from Bloomington Drosophila Stock Center; bdsc.indiana.edu) were injected with a mixture of CRISPR/Cas9 reagents, $100 \mathrm{ng} / \mu$ l guide RNA plasmid and $300 \mathrm{ng} / \mu \mathrm{l}$ repair template DNA vector (University of Cambridge fly facility). Injected male flies were crossed with an in-house Sp/CyO balancer stock for two generations, allowing for the elimination of the vasa::Cas9 carrying $X$ chromosome. Candidate F1 males were genotyped exploiting restriction fragment length polymorphism. All lines were validated by sequencing the region approximately 250 base pairs upstream and downstream of the mutations and sequencing the areas outside the repair templates. In addition, all of the predicted off-target sites were PCRamplified and checked for the presence of any lesions compared with the genomic DNA from the $B L 51323$ line. None of the predicted off-target sites were found to have mutations. To eliminate any other potential off-target mutations introduced during CRISPR, all lines were backcrossed into the $w^{1118}$ control genetic background for six generations.

\section{Restriction fragment length polymorphism assay}

To assess and confirm the presence of the H596F, L283F, R313P, and A348T mutations in the sxc gene, DNA of candidate individual adult flies was extracted using 10-50 $\mu \mathrm{l}$ of DNA extraction buffer containing $10 \mathrm{mM}$ Tris- $\mathrm{HCl} \mathrm{pH} 8,1 \mathrm{mM}$ EDTA, $25 \mathrm{mM} \mathrm{NaCl}$ and $200 \mu \mathrm{g} / \mathrm{ml}$ freshly added Proteinase $\mathrm{K}$ (Roche). The solution was subsequently incubated at $37^{\circ} \mathrm{C}$ for $30 \mathrm{~min}$, followed by inactivation of Proteinase $\mathrm{K}$ at $95{ }^{\circ} \mathrm{C}$ for $3 \mathrm{~min}$, and centrifuged briefly. $1 \mu \mathrm{l}$ of the crude DNA extract was used per $25 \mu \mathrm{l} \mathrm{PCR}$ reaction with the relevant diagnostic primers, using a 2x GoTaq G2 Green premix (Promega). $5 \mu$ of the PCR products were used for restriction fragment length polymorphism assay with the appropriate enzymes, followed by agarose gel electrophoresis of the digested products. Reactions which showed the presence of an undigested full-length PCR product resistant to the expected restriction enzyme cleavage indicated CRISPR/Cas9 gene editing event and were sequenced. Precise incorporation of the repair template into the right position of the genome was confirmed by sequencing a second round of PCR products obtained from potential homozygous CRISPR mutants with mixed diagnostic and line-check primer pairs. Primer sequences are listed in Supplementary Table S1.

\section{Fly stocks and maintenance}

Drosophila stocks and experimental crosses were reared on a standard Drosophila diet (sugar/cornmeal/yeast). An RNAi strain to knockdown sxc (\#18610) and a genetic background control strain (\#60000) were obtained from the Vienna Drosophila Resource Center (VDRC; www.vdrc.at). In-house $s \times C^{H 537 A}$ [50] and UAS-sXc [40] strains, and the generated $s \times C^{H 596 F}$, $s X c^{R 313 P}$, $s \times C^{A 348 T}$, and $s \times C^{L 283 F}$ strains, were crossed into the VDRC $w^{1118}$ control genetic background (\#60000) for six generations. The sxc ${ }^{H 537 A} / C y O$; UAS-sxc strain was assembled using the isogenic strains. Oga ${ }^{K O}$ and $\mathrm{Oga}^{D 133 N}$ lines were also crossed to this background as described earlier [52]. \#60000 was used as isogenic control for the mutant alleles. In the neuromuscular junction analysis of $\mathrm{Sxc}^{\mathrm{R} 313 \mathrm{P}}, \mathrm{sxc}^{\mathrm{A} 348 \mathrm{~T}}$ and $\mathrm{sxc} \mathrm{C}^{\mathrm{L} 283 \mathrm{~F}}$ alleles, the control flies were derived by crossing the flies from the stock used for microinjection (Bloomington Stock: BL51323) and same crossing scheme as that used to derive the SxC ${ }^{\mathrm{R} 313 \mathrm{P}}$, $\mathrm{Sxc}^{\mathrm{A} 348 \mathrm{~T}}$ and $\mathrm{Sxc} \mathrm{C}^{\mathrm{L} 283 \mathrm{~F}}$ homozygotes and eliminate the Cas 9 transgene 
were used. To induce neuronal knockdown and overexpression, a $w^{1118 ;} 2 x G M R$-wIR; elav-Gal4, UAS-Dicer-2 driver strain was used. This strain contains a double insertion of an RNAi construct targeting the gene white specifically in the Drosophila eye (2xGMR-wIR) to suppress pigmentation, as required for an efficient light-off jump response [53,54]. Progeny of the crosses between the driver, RNAi/UAS-sXc and \#60000 strain were used as controls for knockdown and overexpression experiments. All crosses were raised at $25^{\circ} \mathrm{C}, 70 \%$ humidity, and a $12: 12 \mathrm{~h}$ light-dark cycle.

\section{Western blotting}

Protein lysates for Western blotting were prepared from adult male (1-4 days old) fly head samples or 0-16 h embryo collection and snap frozen in liquid nitrogen. Samples were homogenized in lysis buffer containing 2x NuPAGE LDS Sample Buffer, $50 \mathrm{mM}$ Tris- $\mathrm{HCl}(\mathrm{pH} 8.0), 150 \mathrm{mM} \mathrm{NaCl}, 4 \mathrm{mM}$ sodium pyrophosphate, $1 \mathrm{mM}$ EDTA, $1 \mathrm{mM}$ benzamidine, $0.2 \mathrm{mM}$ PMSF, $5 \mu \mathrm{M}$ leupeptin, and $1 \%$ 2-mercaptoethanol. Crude lysates were then incubated for $5 \mathrm{~min}$ at $95{ }^{\circ} \mathrm{C}$, centrifuged at 13000 rpm for $10 \mathrm{~min}$, and supernatants were collected. Pierce $660 \mathrm{~nm}$ protein assay supplemented with Ionic Detergent Compatibility Reagent (Thermo Scientific) was used to determine protein concentration. 20 - $30 \mu \mathrm{g}$ of protein samples were separated on RunBlue $4-12 \%$ gradient gels (Expedeon) using MOPS running buffer, before being transferred onto nitrocellulose membranes. Western blot analysis was carried out with anti-O-GIcNAc (RL2, Abcam, 1:1000) and anti-actin (Sigma, 1:5000) antibodies. Membranes were incubated overnight with selected primary antibodies in $5 \% \mathrm{BSA}$ at $4^{\circ} \mathrm{C}$. Blots were visualized via Li-Cor infrared imaging with Li-Cor secondary antibodies (1:10000) Signal intensities were quantified using ImageStudioLite software. Significance was calculated using one-way ANOVA with Tukey's multiple comparisons test ( $p_{\text {adj }}$ ).

\section{Developmental survival}

Stage 11-16 embryos (25 embryos per vial, 100 per genotype per experiment, $n=3$ ) were cultured at $25{ }^{\circ} \mathrm{C}$ and assessed for lethality by counting the number of pupae and adults derived. Significance was calculated using Student's t-test with Holm-Sidak's correction for multiple comparisons when appropriate.

\section{Light-off jump habituation}

The light-off jump reflex habituation assay was performed as previously described $[48,78]$. Briefly, 3- to 7-day-old individual male flies were subjected to the light-off jump reflex habituation paradigm in two independent 16 -chamber light-off jump habituation systems. Male progeny of the appropriate control genetic background was tested simultaneously on all experimental days. Flies were transferred to the testing chambers without anesthesia. After $5 \mathrm{~min}$ adaptation, a total of 32 flies (16 flies/system) were simultaneously exposed to a series of 100 short $(15 \mathrm{~ms})$ light-off pulses with $1 \mathrm{~s}$ interval. The noise amplitude of wing vibration following every jump response was recorded for $500 \mathrm{~ms}$ after the start of each light-off pulse. A carefully chosen automatic threshold was applied to filter out background noise and distinguish it from jump responses. Data were collected by a custom-made Labview Software (National Instruments). Initial jump responses to light-off pulse decreased with the increasing number of trials and flies were considered habituated when they failed to jump in five consecutive trials (no-jump criterion). Habituation was quantified as the number of trials required to reach the no-jump criterion (Trials To Criterion (TTC)). All experiments were done in triplicates ( $\mathrm{N}=96$ flies). Main effects of genotype on log-transformed TTC values were tested using a linear model regression analysis $(\mathrm{Im})$ in the $R$ statistical software ( $R$ version 3.0.0 (201304-03)) [79] and corrected for the effects of testing day and system. Bonferroni-Holm correction for multiple testing [80] was used to calculate adjusted $p$-values $\left(p_{\text {adj }}\right)$. 


\section{Fatigue assay}

Each genotype that was tested in light-off jump habituation was subsequently subjected to fatigue assay. The fatigue assay was used to evaluate whether the lower TTCs in the rescue experiments were not a result of increased fatigue rather than improved habituation/non-associative learning. The assay was performed as previously described [48]. The interval between light-off pulses was increased to 5 seconds, an intertrial interval that is sufficiently long to prevent habituation. The lightoff pulse was repeated 50 times. Fatigue was concluded when log-transformed TTC values of the rescue were significantly smaller than log-transformed TTC values of the control (based on Im analysis and Bonferroni-Holm correction; $p_{\text {adj }}<0.05$ ).

\section{Analysis of Drosophila neuromuscular junction}

Wandering male L3 larvae were dissected with an open book preparation [81], and fixed in 3.7\% paraformaldehyde for 30 minutes. Larvae were stained overnight at $4{ }^{\circ} \mathrm{C}$ with the primary antibodies against synaptic markers Discs large (anti-dlg1, mouse, 1:25, Developmental Studies Hybridoma Bank) and synaptotagmin (anti-Syt, rabbit, 1:2000, kindly provided by $\mathrm{H}$. Bellen). Secondary antibodies anti-mouse Alexa 488 and anti-rabbit Alexa 568 (Invitrogen) were applied for 2 hours at room temperature (1:500). Projections of type $1 \mathrm{~b}$ neuromuscular junctions (NMJs) at muscle 4 from abdominal segments A2-A4 were assessed. Individual synapses were imaged with a Zeiss Axio Imager Z2 microscope with Apotome and quantified using in-house developed Fiji-compatible macros [82,83]. Anti-dlg1 (4F3 anti-discs large, DSHB, 1:25) labeling was used to analyze NMJ area, length, number of branches and branching points. Anti-Syt (kind gift of Hugo Bellen, 1:2000) labeling was used to analyze the number of synaptic boutons. Secondary antibodies goat antimouse Alexa Fluor 488 (1:200) and goat anti-rabbit Alexa Fluor 568 from Life Technologies were used for visualization. Parameters with a normal distribution (area, length, number of boutons) were compared between the mutants and controls with one-way ANOVA $(p)$ and Tukey's test for multiple comparisons $\left(p_{\text {adj }}\right)$. Parameters without normal distribution (number of branches and branching points) were compared with non-parametric Wilcoxon test ( $p$, single comparisons) and KruskalWallis test with Wilcoxon pairwise test for multiple comparisons $\left(p_{\text {adj }}\right)$ in the R statistical software (R version 3.0.0 (2013-04-03)) [79].

\section{Island assay}

Locomotor behaviour of 3-6 days old male flies was assessed with the island assay as described previously $[84,85]$. Each trial was performed using 15 flies. 3-4 repeats were carried out on each test day, and data was collected on 3 consecutive days. In total, data from 11-16 trials were collected per genotype. The percentage of flies on the island platform over time was plotted and area under curve (AUC) was determined for each run. Groups were compared using one-way ANOVA with Holm-Sidak's multiple comparisons $\left(p_{\text {adj }}\right)$ of means for AUC.

\section{Negative geotaxis test}

The negative geotaxis assay was performed as described previously [86]. The climbing ability of 36 days old male flies was evaluated on groups of 10 animals. Prior to the measurement, flies were transferred into $150 \times 16 \mathrm{~mm}$ transparent plastic test tubes without anesthesia. Test tubes were secured into a frame that allowed for monitoring of climbing behavior of up to 10 vials at once. Upon release, the frame is dropped from a fixed height onto a mouse pad, thereby tapping the flies to the bottom of the tubes. The climbing assay was repeated 4 times for each loaded frame providing data from 4 runs. The experiment was video-recorded with a Nikon D3100 DSLR camera. 
ImageJ/FIJI software was used to analyse the resulting recordings. First, images were converted to an 8-bit grey scale TIFF image sequence (10 frames per second) file format. Backgroundsubtraction and filtering were then applied, and the image pixel values were made binary. The MTrack3 plug-in was used for tracking of flies. Mean climbing speed $(\mathrm{mm} / \mathrm{s})$ was quantified for each genotype in 2nd, 3rd and 4th runs, between 17-89 data points were collected per run. Groups were compared using one-way ANOVA with Tukey's multiple comparisons $\left(p_{\mathrm{adj}}\right)$ of means on mean climbing speed values calculated for each run.

\section{Enrichment analysis}

The O-GlcNAc proteome data was extracted from Selvan et al. (Supplementary dataset 3) [59]. Phenotype annotations of Drosophila gene alleles were extracted from Flybase (Flybase.org, downloaded in April 2016). Human genes implicated in Intellectual disability (ID + ID candidate genes) were extracted from sysid database (https://sysid.cmbi.umcn.nl/, downloaded in April 2016). Enrichment was calculated as follows: $(a / b) /((c-a) /(d-b))$, whereby a =genes in O-GlcNAc proteome and associated with the phenotype term/human ID gene orthologs, $b=$ genes in O-GIcNAc proteome, c=genes associated with the phenotype term/human ID gene orthologs, d=background/all Drosophila genes. Significance was determined using two-sided Fisher's exact test in R [79]. $p$-values were adjusted for multiple testing ( $\left.p_{\text {adj }}\right)$ with Bonferroni-Holm correction [80].

\section{Acknowledgments}

We thank Jennifer Milligan for help with the Western blots and Mehmet Gundogdu for help with the transgenic strains. This work was supported by a ZonMW Vici grant from the Netherlands Organisation for Scientific Research (NWO, No. 09150181910022) to AS, by an ERC consolidator grant (ERC-2017-COG 770244) to ES. We thank the unknown expert referees for helping us to improve the manuscript.

Author Contributions: M.F., V.M., D.M., A.S. and D.M.F.A conceived the study and wrote the manuscript. M.F., V.M., D.M, M.C., I.C. and A.T.F conducted and analyzed the experiments. E.S. supervised M.C.

Competing Interest Statement: The authors declare that there is no conflict of interest.

\section{References}

1. Fujiki R, Hashiba W, Sekine H, Yokoyama A, Chikanishi T, Ito S, et al. GlcNAcylation of histone H2B facilitates its monoubiquitination. Nature [Internet]. 2011;480(7378):557-60. Available from: http://dx.doi.org/10.1038/nature10656

2. Sakabe K, Wang Z, Hart GW. $\beta$-N-acetylglucosamine (O-GlcNAc) is part of the histone code. Proc Natl Acad Sci U S A. 2010;107(46):19915-20.

3. Leturcq M, Lefebvre T, Vercoutter-Edouart AS. O-GIcNAcylation and chromatin remodeling in mammals: An up-to-date overview. Biochem Soc Trans [Internet]. 2017 Apr 15 [cited 2019 Feb 28];45(2):323-38. Available from:

http://www.ncbi.nlm.nih.gov/pubmed/28408473 
4. Ozcan S, Andrali SS, Cantrell JEL. Modulation of transcription factor function by OGlcNAc modification. Vol. 1799, Biochimica et Biophysica Acta - Gene Regulatory Mechanisms. 2010. p. 353-64.

5. Brimble S, Wollaston-Hayden EE, Teo CF, Morris AC, Wells L. The Role of the O-GlcNAc Modification in Regulating Eukaryotic Gene Expression. Curr Signal Transduct Ther [Internet]. 2010 [cited 2019 Feb 28];5(1):12-24. Available from: http://www.ncbi.nlm.nih.gov/pubmed/25484640

6. Zeidan Q, Wang Z, De Maio A, Hart GW. O-GlcNAc cycling enzymes associate with the translational machinery and modify core ribosomal proteins. Mol Biol Cell [Internet]. 2010;21(12):1922-36. Available from:

http://www.pubmedcentral.nih.gov/articlerender.fcgi?artid=2883937\&tool=pmcentrez\&rend ertype $=$ abstract

7. Whelan SA, Dias WB, Thiruneelakantapillai L, Daniel Lane M, Hart GW. Regulation of insulin receptor substrate 1 (IRS-1)/AKT kinase-mediated insulin signaling by O-linked $\beta$ $\mathrm{N}$-acetylglucosamine in 3T3-L1 adipocytes. J Biol Chem [Internet]. 2010;285(8):5204-11. Available from: http://www.jbc.org/content/285/8/5204.long

8. Taub DG, Awal MR, Gabel C V. O-GlcNAc Signaling Orchestrates the Regenerative Response to Neuronal Injury in Caenorhabditis elegans. Cell Rep [Internet]. 2018 Aug 21 [cited 2019 Feb 28];24(8):1931-1938.e3. Available from: http://www.ncbi.nlm.nih.gov/pubmed/30134155

9. Zhang X, Ma L, Qi J, Shan H, Yu W, Gu Y. MAPK/ERK signaling pathway-induced hyperO-GlcNAcylation enhances cancer malignancy. Mol Cell Biochem. 2015;410(1-2):101-10.

10. Yang X, Ongusaha PP, Miles PD, Havstad JC, Zhang F, So WV, et al. Phosphoinositide signalling links O-GIcNAc transferase to insulin resistance. Nature [Internet]. 2008;451(7181):964-9. Available from:

http://www.nature.com.ep.fjernadgang.kb.dk/nature/journal/v451/n7181/full/nature06668.h tml

11. Pekkurnaz G, Trinidad JC, Wang X, Kong D, Schwarz TL. Glucose regulates mitochondrial motility via Milton modification by O-GIcNAc transferase. Cell. 2014;158(1):54-68.

12. Li YH, Liu X, Vanselow JT, Zheng H, Schlosser A, Chiu JC. O-GlcNAcylation of PERIOD regulates its interaction with $\mathrm{CLOCK}$ and timing of circadian transcriptional repression. Taghert PH, editor. PLoS Genet [Internet]. 2019 Jan 31 [cited 2019 Feb 24];15(1):e1007953. Available from: http://dx.plos.org/10.1371/journal.pgen.1007953

13. Bayés A, van de Lagemaat LN, Collins MO, Croning MDR, Whittle IR, Choudhary JS, et al. Characterization of the proteome, diseases and evolution of the human postsynaptic density. Nat Neurosci. 2011;14(1):19-21.

14. Liu F, Iqbal K, Grundke-lqbal I, Hart GW, Gong C-X. O-GlcNAcylation regulates phosphorylation of tau: a mechanism involved in Alzheimer's disease. Proc Natl Acad Sci U S A. 2004;101(29):10804-9. 
15. Liu F, Shi J, Tanimukai H, Gu J, Gu J, Grundke-lqbal I, et al. Reduced O-GlcNAcylation links lower brain glucose metabolism and tau pathology in Alzheimer's disease. Brain. 2009;132(7):1820-32.

16. Levine PM, Galesic A, Balana AT, Mahul-Mellier AL, Navarro MX, De Leon CA, et al. $\alpha-$ Synuclein O-GIcNAcylation alters aggregation and toxicity, revealing certain residues as potential inhibitors of Parkinson's disease. Proc Natl Acad Sci U S A [Internet]. 2019 Jan 29 [cited 2020 Mar 21];116(5):1511-9. Available from:

http://www.ncbi.nlm.nih.gov/pubmed/30651314

17. Grima JC, Daigle JG, Arbez N, Cunningham KC, Zhang K, Ochaba J, et al. Mutant Huntingtin Disrupts the Nuclear Pore Complex. Neuron. 2017;94(1):93-107.e6.

18. Lüdemann N, Clement A, Hans VH, Leschik J, Behl C, Brandt R. O-glycosylation of the tail domain of neurofilament protein $\mathrm{M}$ in human neurons and in spinal cord tissue of a rat model of amyotrophic lateral sclerosis (ALS). J Biol Chem. 2005;280(36):31648-58.

19. Shan X, Vocadlo DJ, Krieger C. Reduced protein O-glycosylation in the nervous system of the mutant SOD1 transgenic mouse model of amyotrophic lateral sclerosis. Neurosci Lett [Internet]. 2012 May 16 [cited 2020 Mar 21];516(2):296-301. Available from: http://www.ncbi.nlm.nih.gov/pubmed/22521585

20. Hanover JA, Wang P. O-GIcNAc cycling shows neuroprotective potential in C. elegans models of neurodegenerative disease. Worm [Internet]. 2013;2(4):e27043. Available from: http://www.pubmedcentral.nih.gov/articlerender.fcgi?artid=3917942\&tool=pmcentrez\&rend ertype=abstract

21. Andres LM, Blong IW, Evans AC, Rumachik NG, Yamaguchi T, Pham ND, et al. Chemical Modulation of Protein O-GIcNAcylation via OGT Inhibition Promotes Human Neural Cell Differentiation. ACS Chem Biol [Internet]. 2017 Aug 18 [cited 2019 Mar 1];12(8):2030-9. Available from: http://www.ncbi.nlm.nih.gov/pubmed/28541657

22. Parween S, Varghese DS, Ardah MT, Prabakaran AD, Mensah-Brown E, Emerald BS, et al. Higher O-GIcNAc levels are associated with defects in progenitor proliferation and premature neuronal differentiation during in-vitro human embryonic cortical neurogenesis. Front Cell Neurosci [Internet]. 2017 Dec 21 [cited 2019 Mar 1];11:415. Available from: http://www.ncbi.nlm.nih.gov/pubmed/29311838

23. Peng P, Wang J, Ding N, Zhou M, Gu Z, Shi Y, et al. Alteration of O-GlcNAcylation affects assembly and axonal transport of neurofilament via phosphorylation. Neurosci Lett [Internet]. 2019 Apr 3 [cited 2019 Feb 28];698:97-104. Available from: http://www.ncbi.nlm.nih.gov/pubmed/30395884

24. Lagerlöf O, Hart GW, Huganir RL. O-GIcNAc transferase regulates excitatory synapse maturity. Proc Natl Acad Sci U S A [Internet]. 2017 Feb 14 [cited 2019 Mar 1];114(7):1684-9. Available from: http://www.ncbi.nlm.nih.gov/pubmed/28143929

25. Bouazzi H, Lesca G, Trujillo C, Alwasiyah MK, Munnich A. Nonsyndromic X-linked intellectual deficiency in three brothers with a novel MED12 missense mutation [c.5922G>T (p.Glu1974His)]. Clin case reports [Internet]. 2015 Jul 1 [cited 2016 Sep 
5];3(7):604-9. Available from: http://onlinelibrary.wiley.com/doi/10.1002/ccr3.301/full

26. Selvan N, George S, Serajee FJ, Shaw M, Hobson L, Kalscheuer V, et al. O-GIcNAc transferase missense mutations linked to $X$-linked intellectual disability deregulate genes involved in cell fate determination and signaling. J Biol Chem. 2018;293(27):10810-24.

27. Vaidyanathan K, Niranjan T, Selvan N, Teo CF, May M, Patel S, et al. Identification and characterization of a missense mutation in the O-linked $\beta-\mathrm{N}$-acetylglucosamine $(\mathrm{O}$ GIcNAc) transferase gene that segregates with X-linked intellectual disability. J Biol Chem. 2017;292(21):8948-63.

28. Willems AP, Gundogdu M, Kempers MJE, Giltay JC, Pfundt R, Elferink M, et al. Mutations in $\mathrm{N}$-acetylglucosamine (O-GlcNAc) transferase in patients with $\mathrm{X}$-linked intellectual disability. J Biol Chem. 2017;292(30):12621-31.

29. Niranjan TS, Skinner C, May M, Turner T, Rose R, Stevenson R, et al. Affected kindred analysis of human $\mathrm{X}$ chromosome exomes to identify novel $\mathrm{X}$-linked intellectual disability genes. PLoS One. 2015;10(2).

30. Pravata VM, Muha V, Gundogdu M, Ferenbach AT, Kakade PS, Vandadi V, et al. Catalytic deficiency of O-GIcNAc transferase leads to X-linked intellectual disability. Proc Natl Acad Sci U S A. 2019;116(30):14961-70.

31. Pravata VM, Gundogdu M, Bartual SG, Ferenbach AT, Stavridis M, Õunap K, et al. A missense mutation in the catalytic domain of O-GIcNAc transferase links perturbations in protein O-GIcNAcylation to X-linked intellectual disability. FEBS Lett [Internet]. 2020 Feb 1 [cited 2020 Mar 21];594(4):717-27. Available from: http://www.ncbi.nlm.nih.gov/pubmed/31627256

32. Pravata VM, Omelková M, Stavridis MP, Desbiens CM, Stephen HM, Lefeber DJ, et al. An intellectual disability syndrome with single-nucleotide variants in O-GlcNAc transferase. European Journal of Human Genetics. Springer Nature; 2020. p. 1-9.

33. Muha V, Williamson R, Hills R, McNeilly AD, McWilliams TG, Alonso J, et al. Loss of CRMP2 O-GlcNAcylation leads to reduced novel object recognition performance in mice. Open Biol [Internet]. 2019 Nov 29 [cited 2020 Mar 21];9(11):190192. Available from: http://www.ncbi.nlm.nih.gov/pubmed/31771416

34. Wheatley EG, Albarran E, White CW, Bieri G, Sanchez-Diaz C, Pratt K, et al. Neuronal OGlcNAcylation Improves Cognitive Function in the Aged Mouse Brain. Curr Biol. 2019;29(20):3359-3369.e4.

35. Joiner CM, Hammel FA, Janetzko J, Walker S. Protein Substrates Engage the Lumen of O-GlcNAc Transferase's Tetratricopeptide Repeat Domain in Different Ways. Biochemistry [Internet]. 2021 Mar 23 [cited 2022 Jan 10];60(11):847-53. Available from: https://pubs.acs.org/doi/pdf/10.1021/acs.biochem.0c00981

36. Llabrés S, Tsenkov MI, MacGowan SA, Barton GJ, Zachariae U. Disease related single point mutations alter the global dynamics of a tetratricopeptide (TPR) $\alpha$-solenoid domain. $J$ Struct Biol [Internet]. 2020 Jan 1 [cited 2021 Jan 8];209(1). Available from: 
https://doi.org/10.1016/j.jsb.2019.107405

37. Gundogdu M, Llabrés S, Gorelik A, Ferenbach AT, Zachariae U, van Aalten DMF. The OGIcNAc Transferase Intellectual Disability Mutation L254F Distorts the TPR Helix. Cell Chem Biol. 2018 May 17;25(5):513-518.e4.

38. Levine ZG, Fan C, Melicher MS, Orman M, Benjamin T, Walker S. O -GlcNAc Transferase Recognizes Protein Substrates Using an Asparagine Ladder in the Tetratricopeptide Repeat (TPR) Superhelix. J Am Chem Soc. 2018;

39. Sinclair D a R, Syrzycka M, Macauley MS, Rastgardani T, Komljenovic I, Vocadlo DJ, et al. Drosophila O-GlcNAc transferase (OGT) is encoded by the Polycomb group (PcG) gene, super sex combs (sxc). Proc Natl Acad Sci U S A. 2009;106(32):13427-32.

40. Mariappa D, Zheng X, Schimpl M, Raimi O, Ferenbach AT, Müller HAJ, et al. Dual functionality of O-GIcNAc transferase is required for Drosophila development. Open Biol. 2015;5(12).

41. Ingham PW. A gene that regulates the bithorax complex differentially in larval and adult cells of Drosophila. Cell. 1984;37(3):815-23.

42. Kim EY, Jeong EH, Park S, Jeong HJ, Edery I, Cho JW. A role for O-GIcNAcylation in setting circadian clock speed. Genes Dev. 2012 Mar 1;26(5):490-502.

43. Colombo J, Mitchell DW. Infant visual habituation. Neurobiol Learn Mem. 2009;92(2):22534.

44. Rankin $\mathrm{CH}$, Abrams T, Barry RJ, Bhatnagar S, Clayton D, Colombo J, et al. Habituation Revisited: An Updated and Revised Description of the Behavioral Characteristics of Habituation. Neurobiol Learn Mem [Internet]. 2009 Sep 6;92(2):135-8. Available from: http://www.ncbi.nlm.nih.gov/pmc/articles/PMC2754195/

45. Barron HC, Vogels TP, Behrens TE, Ramaswami M. Inhibitory engrams in perception and memory. Proc Natl Acad Sci [Internet]. 2017 Jun 13 [cited 2019 Mar 2];114(26):201701812. Available from: http://www.ncbi.nlm.nih.gov/pubmed/28611219

46. McDiarmid TA, Bernardos AC, Rankin $\mathrm{CH}$. Habituation is altered in neuropsychiatric disorders-A comprehensive review with recommendations for experimental design and analysis. Neurosci Biobehav Rev [Internet]. 2017 Sep [cited 2019 Mar 2];80:286-305. Available from: http://www.ncbi.nlm.nih.gov/pubmed/28579490

47. Stessman HAF, Xiong B, Coe BP, Wang T, Hoekzema K, Fenckova M, et al. Targeted sequencing identifies 91 neurodevelopmental-disorder risk genes with autism and developmental-disability biases. Nat Genet. 2017;49(4):515-26.

48. Fenckova M, Blok LER, Asztalos L, Goodman DP, Cizek P, Singgih EL, et al. Habituation Learning Is a Widely Affected Mechanism in Drosophila Models of Intellectual Disability and Autism Spectrum Disorders. Biol Psychiatry. 2019;

49. Chou VT, Johnson SA, Van Vactor D. Synapse development and maturation at the 
drosophila neuromuscular junction. Neural Dev [Internet]. 2020 Aug 2 [cited 2021 Dec 13];15(1):1-17. Available from:

https://neuraldevelopment.biomedcentral.com/articles/10.1186/s13064-020-00147-5

50. Mariappa D, Ferenbach AT, Daan MFVA. Effects of hypo-o-glcnacylation on drosophila development. J Biol Chem. 2018;293(19):7209-21.

51. Engel JE, Wu CF. Altered habituation of an identified escape circuit in Drosophila memory mutants. J Neurosci [Internet]. 1996;16(10):3486-99. Available from:

http://www.ncbi.nlm.nih.gov/pubmed/8627381

52. Muha V, Fenckova M, Ferenbach AT, Catinozzi M, Eidhof I, Storkebaum E, et al. OGlcNAcase contributes to cognitive function in Drosophila. J Biol Chem [Internet]. 2020 Feb 24 [cited 2020 Mar 5];jbc.RA119.010312. Available from: http://www.ncbi.nlm.nih.gov/pubmed/32094227

53. Willemsen MH, Nijhof B, Fenckova M, Nillesen WM, Bongers EMHF, Castells-Nobau A, et al. GATAD2B loss-of-function mutations cause a recognisable syndrome with intellectual disability and are associated with learning deficits and synaptic undergrowth in Drosophila. J Med Genet. 2013;50(8):507-14.

54. van Bon BWM, Oortveld MAW, Nijtmans LG, Fenckova M, Nijhof B, Besseling J, et al. CEP89 is required for mitochondrial metabolism and neuronal function in man and fly. Hum Mol Genet. 2013;22(15):3138-51.

55. Esmaeeli-Nieh S, Fenckova M, Porter IM, Motazacker MM, Nijhof B, Castells-Nobau A, et al. BOD1 Is Required for Cognitive Function in Humans and Drosophila. Kooy RF, editor. PLOS Genet [Internet]. 2016 May 11;12(5):e1006022. Available from: https://dx.plos.org/10.1371/journal.pgen.1006022

56. Haddad DM, Vilain S, Vos M, Esposito G, Matta S, Kalscheuer VM, et al. Mutations in the Intellectual Disability Gene Ube2a Cause Neuronal Dysfunction and Impair ParkinDependent Mitophagy. Mol Cell [Internet]. 2013 Jun 27 [cited 2019 Mar 2];50(6):831-43. Available from: https://www.sciencedirect.com/science/article/pii/S1097276513002931

57. Zweier C, de Jong EK, Zweier M, Orrico A, Ousager LB, Collins AL, et al. CNTNAP2 and NRXN1 Are Mutated in Autosomal-Recessive Pitt-Hopkins-like Mental Retardation and Determine the Level of a Common Synaptic Protein in Drosophila. Am J Hum Genet. 2009;85(5):655-66.

58. Zhang YQ, Bailey AM, Matthies HJG, Renden RB, Smith MA, Speese SD, et al. Drosophila fragile $x$-related gene regulates the MAP1B homolog Futsch to control synaptic structure and function. Cell [Internet]. 2001 Nov 30 [cited 2019 Mar 2];107(5):591-603. Available from: https://www.sciencedirect.com/science/article/pii/S009286740100589X

59. Selvan N, Williamson R, Mariappa D, Campbell DG, Gourlay R, Ferenbach AT, et al. A mutant O-GlcNAcase enriches Drosophila developmental regulators. Nat Chem Biol. 2017;13(8):882-7.

60. Barron HC, Vogels TP, Behrens TE, Ramaswami M. Inhibitory engrams in perception and 
memory. Proc Natl Acad Sci. 2017;201701812.

61. Ardiel EL, McDiarmid TA, Timbers TA, Lee KCY, Safaei J, Pelech SL, et al. Insights into the roles of CMK-1 and OGT-1 in interstimulus interval-dependent habituation in Caenorhabditis elegans. Proceedings Biol Sci [Internet]. 2018 Nov 14 [cited 2019 Mar 2];285(1891):20182084. Available from: http://rspb.royalsocietypublishing.org/lookup/doi/10.1098/rspb.2018.2084

62. Keshishian H, Broadie K, Chiba A, Bate M. The drosophila neuromuscular junction: a model system for studying synaptic development and function. Annu Rev Neurosci [Internet]. 1996;19:545-75. Available from: http://eutils.ncbi.nlm.nih.gov/entrez/eutils/elink.fcgi?dbfrom=pubmed\&id=8833454\&retmod e=ref\&cmd=prlinks\%5Cnpapers3://publication/doi/10.1146/annurev.ne.19.030196.002553

63. Sanhueza M, Zechini L, Gillespie T, Pennetta G. Gain-of-function mutations in the ALS8 causative gene VAPB have detrimental effects on neurons and muscles. Biol Open [Internet]. 2014 Jan 15 [cited 2019 Mar 4];3(1):59-71. Available from: http://www.ncbi.nlm.nih.gov/pubmed/24326187

64. Harris KP, Akbergenova Y, Cho RW, Baas-Thomas MS, Littleton JT. Shank modulates postsynaptic wnt signaling to regulate synaptic development. J Neurosci [Internet]. 2016 [cited 2019 Mar 4];36(21):5820-32. Available from:

http://www.ncbi.nlm.nih.gov/pubmed/27225771

65. Zhang X, Rui M, Gan G, Huang C, Yi J, Lv H, et al. Neuroligin 4 regulates synaptic growth via the bone morphogenetic protein (BMP) signaling pathway at the Drosophila neuromuscular junction. J Biol Chem [Internet]. 2017 Nov 3 [cited 2019 Mar 4];292(44):17991-8005. Available from: http://www.ncbi.nlm.nih.gov/pubmed/28912273

66. McCabe BD, Hom S, Aberle H, Fetter RD, Marques G, Haerry TE, et al. Highwire regulates presynaptic BMP signaling essential for synaptic growth. Neuron [Internet]. 2004 Mar 25 [cited 2019 Mar 4];41(6):891-905. Available from: https://www.sciencedirect.com/science/article/pii/S089662730400073X

67. Ataman B, Ashley J, Gorczyca M, Ramachandran P, Fouquet W, Sigrist SJ, et al. Rapid Activity-Dependent Modifications in Synaptic Structure and Function Require Bidirectional Wnt Signaling. Neuron [Internet]. 2008 Mar 13 [cited 2019 Mar 4];57(5):705-18. Available from: http://www.ncbi.nlm.nih.gov/pubmed/18341991

68. Piccioli ZD, Littleton JT. Retrograde BMP signaling modulates rapid activity-dependent synaptic growth via presynaptic lim kinase regulation of Cofilin. J Neurosci [Internet]. 2014 Mar 19 [cited 2019 Mar 4];34(12):4371-81. Available from: http://www.ncbi.nlm.nih.gov/pubmed/24647957

69. Choi BJ, Imlach WL, Jiao W, Wolfram V, Wu Y, Grbic M, et al. Miniature Neurotransmission Regulates Drosophila Synaptic Structural Maturation. Neuron [Internet]. 2014 May 7 [cited 2019 Mar 4];82(3):618-34. Available from: http://www.ncbi.nlm.nih.gov/pubmed/24811381

70. Ji S, Kang JG, Park SY, Lee J, Oh YJ, Cho JW. O-GIcNAcylation of tubulin inhibits its 
polymerization. Amino Acids [Internet]. 2011 Mar 28 [cited 2019 Mar 4];40(3):809-18.

Available from: http://www.ncbi.nlm.nih.gov/pubmed/20665223

71. Budnik V. Synapse maturation and structural plasticity at Drosophila neuromuscular junctions. Curr Opin Neurobiol [Internet]. 1996 [cited 2021 Dec 14];6(6):858-67. Available from: https://pubmed.ncbi.nlm.nih.gov/9000022/

72. lyer SP, Hart GW. Roles of the TPR domain in O-GIcNAc transferase (OGT) Targeting and protein substrate specificity. J Biol Chem. 2003;

73. Rafie K, Raimi O, Ferenbach AT, Borodkin VS, Kapuria V, van Aalten DMF. Recognition of a glycosylation substrate by the O-GIcNAc transferase TPR repeats. Open Biol. 2017;

74. Lazarus MB, Jiang J, Kapuria V, Bhuiyan T, Janetzko J, Zandberg WF, et al. HCF-1 is cleaved in the active site of O-GlcNAc transferase. Science (80-) [Internet]. 2013 [cited 2021 Jan 10];342(6163):1235-9. Available from: /pmc/articles/PMC3930058/?report=abstract

75. Yeung K, Boija A, Karlsson E, Holmqvist PH, Tsatskis Y, Nisoli I, et al. Atrophin controls developmental signaling pathways via interactions with Trithorax-like. Elife [Internet]. 2017 Mar 22 [cited 2022 Jan 10];6. Available from: https://pubmed.ncbi.nlm.nih.gov/28327288/

76. Port F, Chen HM, Lee T, Bullock SL. Optimized CRISPR/Cas tools for efficient germline and somatic genome engineering in Drosophila. Proc Natl Acad Sci U S A. 2014;111(29).

77. Van Den Ent F, Löwe J. RF cloning: A restriction-free method for inserting target genes into plasmids. J Biochem Biophys Methods. 2006;67(1):67-74.

78. Kramer JM, Kochinke K, Oortveld MAW, Marks H, Kramer D, de Jong EK, et al. Epigenetic regulation of learning and memory by Drosophila EHMT/G9a. PLoS Biol. $2011 ; 9(1)$.

79. R Development Core Team R. R: A Language and Environment for Statistical Computing [Internet]. Vol. 1, R Foundation for Statistical Computing. 2011. p. 409. Available from: http://www.r-project.org

80. Sture Holm. A Simple Sequentially Rejective Multiple Test Procedure. Scand J Stat. 1979;6(2):65-70.

81. Brent JR, Werner KM, McCabe BD. Drosophila Larval NMJ Dissection. J Vis Exp. 2009;

82. Nijhof B, Castells-Nobau A, Wolf L, Scheffer-de Gooyert JM, Monedero I, Torroja L, et al. A New Fiji-Based Algorithm That Systematically Quantifies Nine Synaptic Parameters Provides Insights into Drosophila NMJ Morphometry. PLoS Comput Biol. 2016;12(3).

83. Castells-Nobau A, Nijhof B, Eidhof I, Wolf L, Scheffer-de Gooyert JM, Monedero I, et al. Two Algorithms for High-throughput and Multi-parametric Quantification of \&lt;em\&gt;Drosophila\&It;/em\&gt; Neuromuscular Junction Morphology. J Vis Exp. 2017;

84. Schmidt I, Thomas S, Kain P, Risse B, Naffin E, Klämbt C. Kinesin heavy chain function in 
Drosophila glial cells controls neuronal activity. J Neurosci. 2012;32(22):7466-76.

85. Eidhof I, Fenckova M, Elurbe DM, van de Warrenburg B, Nobau AC, Schenck A. Highthroughput analysis of locomotor behavior in the Drosophila island assay. J Vis Exp. 2017;2017(129).

86. Niehues S, Bussmann J, Steffes G, Erdmann I, Köhrer C, Sun L, et al. Impaired protein translation in Drosophila models for Charcot-Marie-Tooth neuropathy caused by mutant tRNA synthetases. Nat Commun. 2015;6. 


\section{Figure Legends}

Figure 1. Catalytic activity of O-GICNAc transferase in neurons is required for habituation learning

Jump responses were induced by 100 light-off pulses with $1 \mathrm{~s}$ interval between pulses. The jump response represents the \% of jumping flies in each light-off trial. The mean number of trials that flies needed to reach the no-jump criterion (Trials To Criterion, TTC) \pm SEM is also shown.

(A) Defective habituation of $s x c^{H 537 A /+}$ flies $(N=59$, mean TTC \pm SD: $12.8 \pm 6.7 p=0.001$, in red) compared to their respective genetic background control flies $(+/+$, mean TTC \pm SD: $4.4 \pm 0.9, \mathrm{~N}=61$, in blue). ${ }^{* *} p<0.01$, based on Im analysis.

(B) Habituation defect of $s x c^{H 537 A /+}$ flies $\left(N=72\right.$, mean TTC $\pm S D$ : $15.5 \pm 7.8 p_{\text {adj }}=0.045$, in red) is corrected by removing one Oga allele in $s x c^{H 537 A /+}$; Oga ${ }^{K O /+}$ flies $(N=70$, mean TTC \pm SD: $10.1 \pm 5.9, p_{\text {adj }}=0.024$, in cyan) to the level of control flies $(N=72$, mean TTC \pm SD: $9.4 \pm$ $3.5, p_{\text {adj }}=0.677$, in blue $)$. Habituation of Oga ${ }^{K O /+}$ flies $(\mathrm{N}=76$, mean TTC \pm SD: $17.1 \pm 10.4$, in purple) is slower but not significantly different from the control flies ( $p_{\text {adj }}=0.467$ ).

(C) Defective habituation of elav-Gal4>UAS-sxc flies ( $N=55$, mean TTC \pm SD: $12.8 \pm 4, p_{\text {adj }}=$ $3.89 \times 10^{-12}$, in dark blue) compared to control elav-Gal4/+ flies $(\mathrm{N}=38$, mean TTC \pm SD: 2.6 \pm 1.3 in light blue).

(D) Habituation defect of $s \times C^{H 537 A /+}$; elav-Gal4/+ flies ( $\mathrm{N}=40$, mean TTC $\pm \mathrm{SD}: 14.6 \pm 6, p_{\text {adj }}=$ $4.92 \times 10^{-12}$, in red) is corrected by selective expression of UAS-sXC in neurons ( sxc $^{\mathrm{H} 537 \mathrm{~A} /+}$; elav-Gal4>UAS-sxc, $\mathrm{N}=43$, mean TTC $\pm \mathrm{SD}: 4.2 \pm 1.3, p_{\text {adj }}=8.94 \times 10^{-7}$, in green), to the level of the genetic background control flies $\left(+/+, N=65\right.$, mean TTC \pm SD: $4.4 \pm 0.9$, $p_{\text {adj }}=$ 0.68 , in blue). ${ }^{*} p_{\text {adj }}<0.1,{ }^{* * *} p_{\text {adj }}<0.001$, n.s. not significant, based on Im analysis with Bonferroni-Holm correction for multiple comparisons. A complete list of $p$-values and summary statistics is provided in Table $\mathbf{S 3}$.

Figure 2. Both reduced and increased O-GIcNAc transferase activity in neurons cause defects in synaptic morphology

Data presented as individual data points with mean \pm SD.

(A) NMJs on muscle 4 of $s x c^{H 537 A /+}$ larvae have a significantly higher number of synaptic boutons $(\mathrm{N}=29$, in red) as compared to their genetic background control $(+/+, \mathrm{N}=24, p=$ 0.009 , in blue, ) but not significantly different NMJ length $(p=0.872)$, area $(p=0.314)$ and perimeter $(p=0.935) .{ }^{* *} p<0.01$, based on one-way ANOVA.

(B) Elav-Gal4>UAS-sxc larvae have a significantly lower number of boutons $(\mathrm{N}=29$, in dark blue) compared to their respective background controls elav-Gal4/+ $\left(\mathrm{N}=30, p_{\text {adj }}=2.1 \times 10^{-}\right.$ ${ }^{4}$, in light blue $)$ and $U A S-s x C /+\left(N=26, p_{\text {adj }}=0.009\right.$, in grey $)$, significantly reduced NMJ length $\left(p_{\text {adj } / \text { elav-Gal } 4}=0.013, p_{\text {adj } j \text { UAS-sxc }}=0.02\right)$ and a smaller NMJ perimeter $\left(p_{\text {adj } / U A S-s x c}=\right.$ $0.008)$.

(C) Neuron-selective expression of sxc in $s \times c^{H 537 A /+}$ larvae partially corrects the bouton numbers ( $s x c^{H 537 A /+}$; elav-Gal4>UAS-sXc $\left(\mathrm{N}=29\right.$, in green)) compared to $s \times c^{H 537 A /+}$, UASsxc/+ larvae $\left(\mathrm{N}=28, p_{\text {adj }}=0.124\right.$, in red $)$ and compared to control $\left(\mathrm{N}=28, p_{\text {adj }}=0.8\right.$, in blue). $s \times C^{H 537 A /+}$; elav-Gal4>UAS-sXc larvae do not show any change in NMJ length compared to control $\left(+/+, N=28, p_{\text {adj }}=0.974\right)$ and $s \times c^{H 537 A /+}$, UAS-sxc/+ larvae $\left(p_{\text {adj }}=\right.$ $0.935)$. Also NMJ area was not changed ( $\left.p_{\text {adj/control }}=0.849, p_{\text {adj } / \text { sxcH537A; UAS-sxc/ }+}=0.691\right)$. 
SxCH537A/+; elav-Gal4>UAS-sxc larvae show significantly increased NMJ perimeter as compared to control larvae ( $\left.p_{\text {adj }}=0.047\right)$ but not $s \times c^{H 537 A /+}$, UAS-s $x c /+$ larvae $\left(p_{\text {adj }}=0.995\right)$.

(D) Number of synaptic boutons $\left(p_{\text {adj }}=0.003\right)$, NMJ length $\left(p_{\text {adj }}=0.01\right)$, area $\left(p_{\text {adj }}=0.028\right)$ and

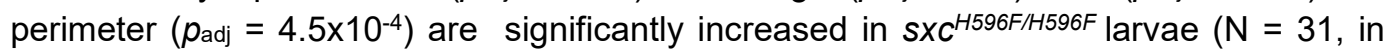
brown) compared to the genetic background control larvae $(+/+, N=28$, in blue) and partially normalized in the sxc $\mathrm{H}^{\mathrm{5} 96 \mathrm{~F} / \mathrm{H} 596 \mathrm{~F}}$; Oga $\mathrm{KO}^{\mathrm{K} O}$ larvae $(\mathrm{N}=30$, in cyan, boutons: $p_{\text {adj } / \text { sxcH596F }}=0.011, p_{\text {adj } / \text { control }}=0.923$; length: $p_{\text {adj } / \text { sxcH596A }}=0.896, p_{\text {adj } / \text { control }}=0.001 ;$ area: $p_{\text {adj/sxcH596A }}=0.501, p_{\text {adj/control }}=0.431$; perimeter: $\left.p_{\text {adj } / \text { sxcH596A }}=0.08, p_{\text {adj } / \text { control }}=0.26\right)$. None of the parameters is significantly affected in the Oga ${ }^{K O}$ larvae $\left(\mathrm{N}=30\right.$, in purple; Oga ${ }^{K O}$ experiments were performed simultaneously and first published here [52] with significantly increased bouton counts $(p<0.05)$ without multiple testing correction). ${ }^{*} p_{\text {adj }}<0.05,{ }^{* *} p_{\text {adj }}$ $<0.01,{ }^{* * *} p_{\text {adj }}<0.001$, based on one-way ANOVA with Tukey's multiple comparisons test. A complete list of $p$-values and summary statistics is provided in Table S3.

(A'- D') Representative NMJs of wandering third instar larvae labeled with anti-discs large 1 (D/g, magenta) and anti-synaptotagmin (Syt, green). When appropriate, type $1 \mathrm{~b}$ synapses are distinguished from other synapses with white arrow. Scale bar, $20 \mu \mathrm{m}$. The quantitative parameter values of the representative images: $\left(\mathbf{A}^{\prime}\right)\left(+/+\mid s x c^{H 537 A /+}\right)$ : \#Boutons (31 | 39), Length (103.7 | 137.8), Area (374.4 | 369.4), Perimeter (245.5 | 306.7) (B') (elav-Gal4/+ | UAS-sxc/+ | ElavGal4>UAS-sxc): \#Boutons (45 | $37 \mid 28)$, Length (160.0 | 137.5 | 93.3), Area (480.4 | 478.4 | 363.9),

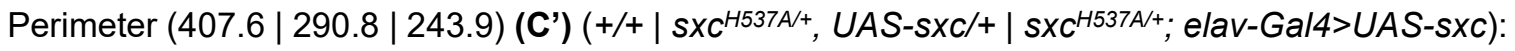
\#Boutons (27 | $34 \mid$ 31), Length (107.6 | 144.9 | 114.9), Area (430.6 | 464.7 | 361.7), Perimeter

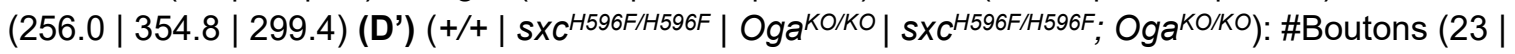
43 | 35 | 30), Length (111.5 | 205.6 | 122.9 | 142.5), Area (351.7 | 691.7 | 417.8 | 377.6), Perimeter (245.6 | 544.0 | 274.1 | 318.9).

Figure 3. Developmental, locomotor and biochemical characterization of $\operatorname{sxc}^{R 313 P}, \operatorname{sxc}^{A 348 T}$ and $s \times c^{L 283 F}$ flies

(A) Control, $s x c^{R 313 P}, s x c^{A 348 T}$ and $s x c^{L 283 F}$ embryos (stage 11-16, 80-100 per experiment) were transferred to fresh food at $25^{\circ} \mathrm{C}$, and the numbers of pupae formed and adults eclosed were counted. Development from embryo to pupae or from pupae to adulthood was not significantly affected in $s x c^{R 313 P}$ (pupae: $\mathrm{N}=4$ repeats, $p=0.9$, adults: $\mathrm{N}=3, p=0.29$, in red), $s x c^{A 348 T}$ (pupae: $N=3, p=0.152$, adults: $N=3, p=0.345$, in blue) and $s x c^{L 283 F}$ mutants (pupae: $\mathrm{N}=4, p=0.108$, adults: $\mathrm{N}=3, p=0.727$, in green). Data presented as individual data points with mean $\pm \mathrm{SD}$. P-values are based on Student's t-test.

(B) Flight escape performance was assessed in the island assay. 15 flies per measurement were thrown on a white platform surrounded with water. Data was collected over 3 days of measurement (Control: $\mathrm{N}=23, \operatorname{sxc}^{R 313 P}: \mathrm{N}=16$, sxc $^{A 348 T}: \mathrm{N}=15$ and $s \times c^{L 283 F}: \mathrm{N}=14$ repeats). Floating bars depict mean $\pm S D$ area under curve (AUC), a parameter that is derived from data plotted as \% flies on the platform over time. One-way ANOVA with Tukey's multiple comparisons was used to compare the mean AUC between genotypes. Flight escape performance of $s X c^{R 313 P}, s X c^{A 348 T}$ and $s X c^{L 283 F}$ flies revealed no defects in locomotion or fitness.

(C) Climbing locomotor behaviour was assessed based on the climbing speed $(\mathrm{mm} / \mathrm{s})$ in an automated negative geotaxis assay. The $s \times c^{R 313 P /+}$ and $s \times c^{R 313 P}(\mathrm{~N}=9)$ flies showed reduced climbing speed compared to background control $(N=9)$ indicating locomotor dysfunction. $s x c^{A 348 T}, s x c^{L 283 F}, s x c^{H 537 A}$ and $s x c^{H 596 F}$ flies ( $N=9$ for all genotypes) did not show significantly reduced climbing speed. Data presented as mean $\pm \mathrm{SD}$. * $\mathrm{p}_{\text {adj }}<0.05$ 
based on one-way ANOVA with Tukey's multiple comparisons of mean climbing speed. A complete list of $p$-values and summary statistics is provided in Table $\mathbf{S 2}$.

(D) Western blot on head samples from 1-4 days old male adult Drosophila indicate no significant alteration in the level of protein O-GIcNAcylation in $s X C^{R 313 P}, s X c^{A 348 T}$ and $s X c^{L 283 F}$ samples compared to the genetic background controls, while the homozygous Oga ${ }^{K O}$ allele caused an increase of O-GlcNAcylation. Western blot was probed with a monoclonal antiO-GlcNAc antibody (RL2).

(E) Quantification of O-GIcNAcylated proteins revealed that protein O-GIcNAcylation in $s X c^{R 313 P}, s X C^{A 348 T}$ and $s X C^{L 283 F}$ flies remain at a similar level as in the control samples. Data presented as mean $\pm S D$. * padj $=0.035$, based on one-way ANOVA with Tukey's multiple comparisons test, $n=3$ for all lines. A complete list of $p$-values and summary statistics is provided in Table S3.

\section{Figure 4. Assessment of $s \times c^{R 313 P}$ and $s \times c^{A 348 T}$ flies in habituation learning}

Jump responses were induced by 100 light-off pulses with $1 \mathrm{~s}$ interval between pulses. The jump response represents the \% of jumping flies in each light-off trial. The mean number of trials that flies needed to reach the no-jump criterion (Trials To Criterion, TTC) \pm SEM is also shown.

(A) Deficient habituation of $s \times c^{R 313 P /+}$ flies $\left(\mathrm{N}=73, \mathrm{p}_{\mathrm{adj}}=6.18 \times 10^{-6}\right.$, in red) compared to their respective genetic background controls (control, $\mathrm{N}=65$, in blue).

(B) Deficient habituation of $s x c^{A 348 T / A 348 T}$ flies $\left(N=76, p_{\text {adj }}=2.1 \times 10^{-14}\right.$, in brown) and no significant habituation deficit of $s \times c^{A 348 T /+}$ flies $\left(N=72, p_{\text {adj }}=0.095\right.$, in red) compared to the genetic background control (control, $\mathrm{N}=65$, in blue).

(C) Deficient habituation of $s \times c^{R 313 P /+}$ flies $\left(\mathrm{N}=81\right.$, $\mathrm{padj}=2.63 \times 10^{-6}$, in red $)$ is restored in $s \times c^{R 313 P /+} ;$ Oga $^{K O /+}$ flies $\left(\mathrm{N}=53\right.$, padj $=4.89 \times 10^{-5}$, in cyan $)$.

(D) Deficient habituation of $s \times c^{A 348 T / A 348 T}$ flies $\left(\mathrm{N}=79\right.$, $p_{\text {adj }}=8.84 \times 10^{-10}$, in brown) is restored in $s \times c^{A 348 T / A 348 T}$; Oga ${ }^{K O / K O}$ flies ( $\mathrm{N}=62$, padj $=1.07 \times 10^{-4}$, in cyan).

(E) Deficient habituation of $s \times c^{R 313 P /+}$ flies $\left(\mathrm{N}=81, p_{\text {adj }}=2.63 \times 10^{-6}\right.$, in red) is restored in $s \times C^{R 313 P /+} ; O a^{D 133 N /+}$ flies ( $\mathrm{N}=64$, padj $=9.09 \times 10^{-6}$, in cyan $)$.

(F) Deficient habituation of $s \times c^{A 348 T / A 348 T}$ flies $\left(\mathrm{N}=79\right.$, $p_{\text {adj }}=8.84 \times 10^{-10}$, in brown) is restored in sXc ${ }^{A 348 T / A 348 T}$; Oga ${ }^{D 133 N / D 133 N}$ flies $\left(N=56, p_{a d j}=3.74 \times 10^{-7}\right.$, in cyan). ${ }^{* * *} p_{a d j}<0.001$, n.s. not significant, based on Im analysis with Bonferroni-Holm correction for multiple comparisons. A complete list of p-values and summary statistics is provided in Table $\mathbf{S} 3$.

Figure 5. Synaptic morphology of $s \times c^{R 313 P}, s x c^{A 348 T}$ and $s \times c^{L 283 F}$

Data presented as individual data points with mean \pm SD.

(A) NMJs on muscle 4 of $s X c^{R 313 P}, s X c^{A 348 T}$ and $s X c^{L 283 F}$ larvae have a significantly higher number of synaptic boutons $\left(s X C^{R 313 P}: \mathrm{N}=21, \mathrm{p}=6.8 \times 10^{-5}\right.$, in red; $s \times c^{A 348 T}: \mathrm{N}=21, \mathrm{p}=$ 0.011 , in blue; $\left.s X c^{L 283 F}: \mathrm{N}=20, \mathrm{p}=0.0225\right)$ compared to the control $(+/+, \mathrm{N}=42$, in grey). sxc ${ }^{R 313 P}$ larvae have also significantly higher NMJ length $(p=0.0125)$ and perimeter $(p=$ 0.001). ). ${ }^{*} p_{\text {adj }}<0.05,{ }^{* *} p_{\text {adj }}<0.01,{ }^{* * *} p_{\text {adj }}<0.001$, based on one-way ANOVA with Tukey's multiple comparisons test. A complete list of $p$-values and summary statistics is provided in Table S3.

(A') Representative NMJs of wandering third instar larvae labeled with anti-discs large 1 (D/g, magenta) and anti-synaptotagmin (Syt, green). When appropriate, type $1 \mathrm{~b}$ synapses are

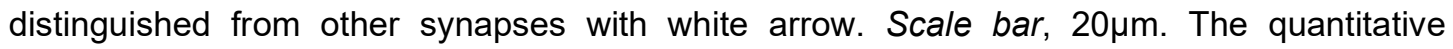
parameter values of the representative images $\left(+/+\left|s x c^{R 313 P}\right| s x c^{A 348 T} \mid s x c^{L 283 F}\right)$ : \#Boutons 
bioRxiv preprint doi: https://doi.org/10.1101/2022.01.10.475726; this version posted January 12,2022 . The copyright holder for this preprint (which was not certified by peer review) is the author/funder, who has granted bioRxiv a license to display the preprint in perpetuity. It is made available under aCC-BY-NC-ND 4.0 International license.

(20 | 36 | 29 | 37), Length (84.5 | 113.8 | 107.4 | 128), Area (399 | 447.9 | 360.9 | 349.9), Perimeter (254.2 | 329.5 | 313.8 | 302.4). 
bioRxiv preprint doi: https://doi.org/10.1101/2022.01.10.475726; this version posted January 12, 2022. The copyright holder for this preprint (which was not certified by peer review) is the author/funder, who has granted bioRxiv a license to display the preprint in perpetuity. It is made Figure 1
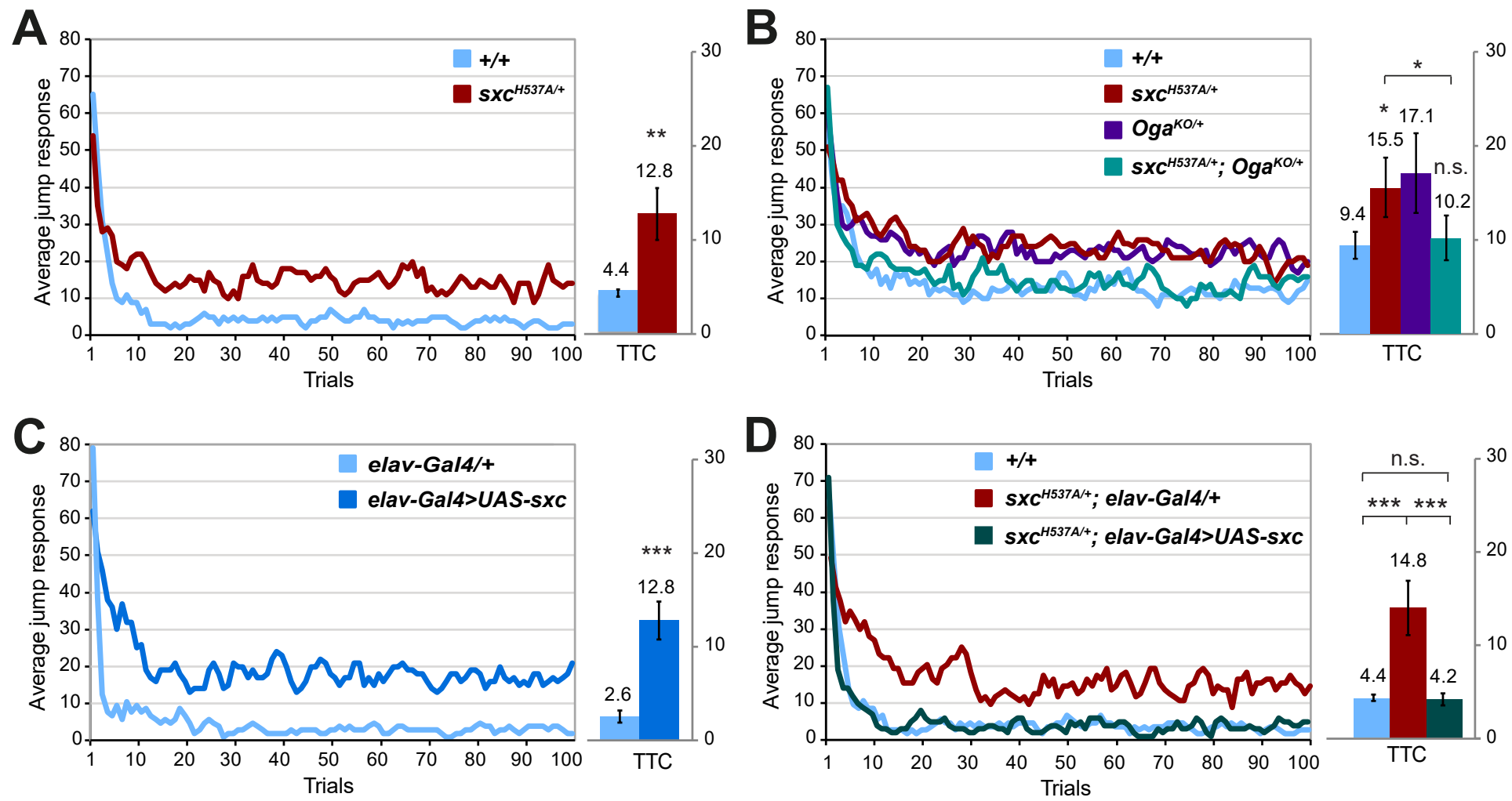


\section{Figure 2}

AioRxiv preprint doi: https://doi.org/10.1101/2022.01.10.475726; this version posted January 12, 2022. The copyright holder for this preprint (Which \#ass not certified by peer revieww is the author/funder, whohas granted bioRxiv a license to display the preprint in perpetuity. It is made
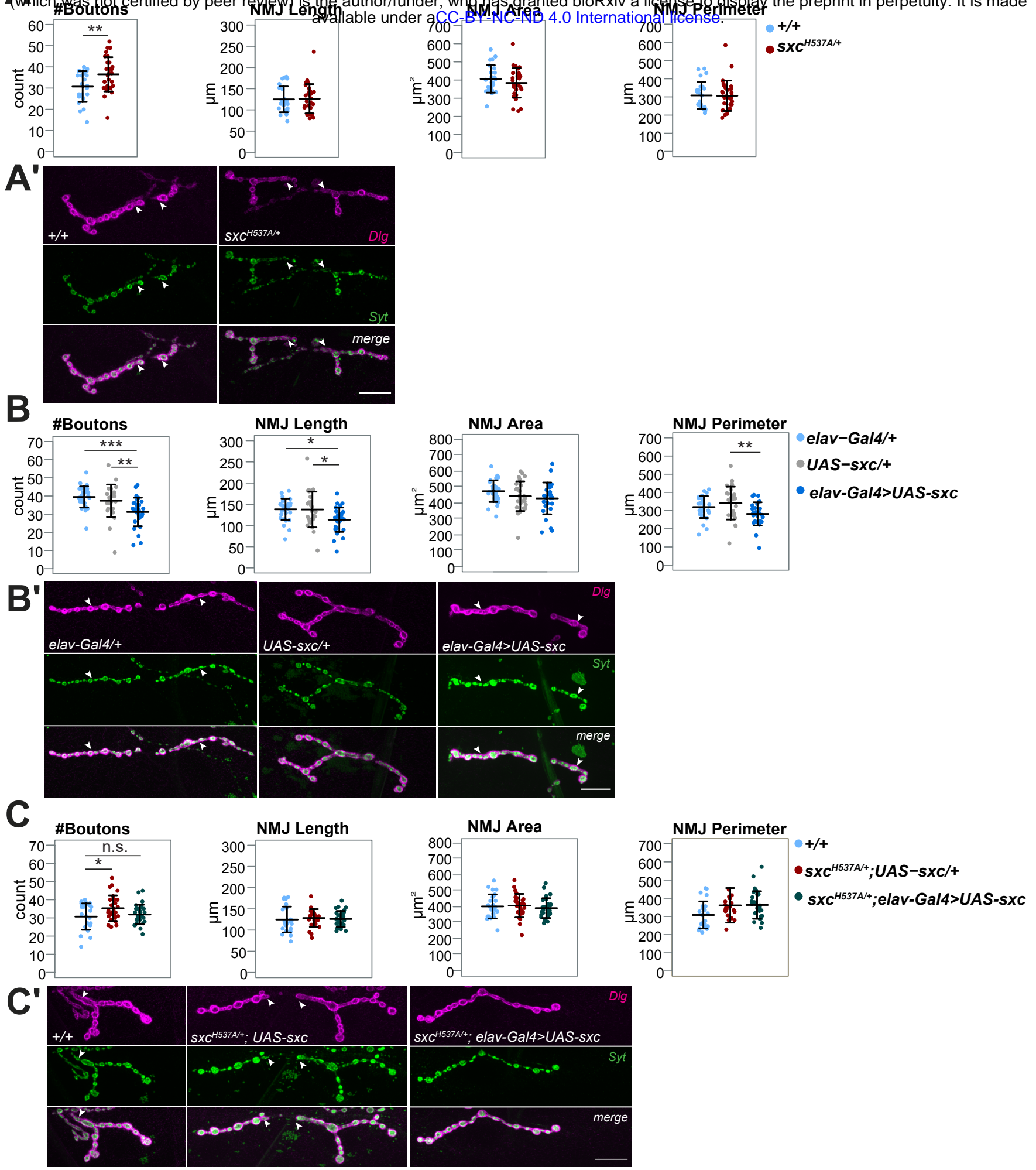

D
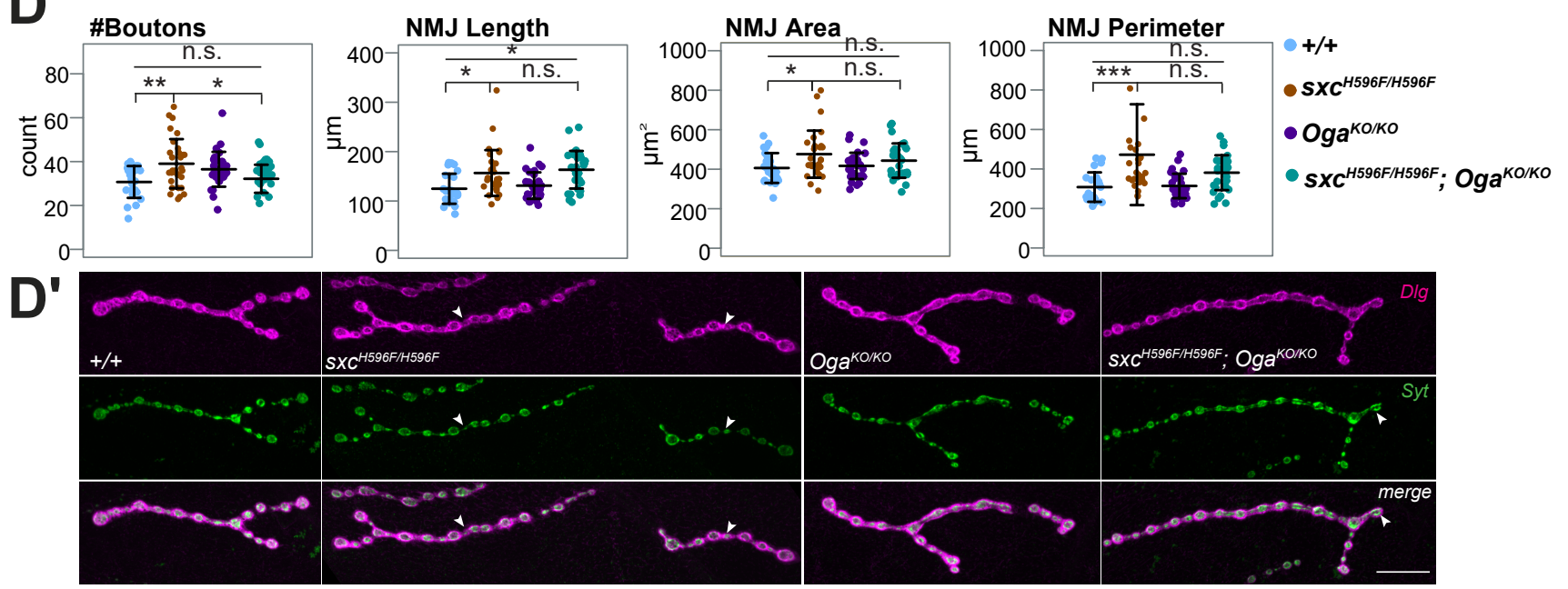
bioRxiv preprint doi: https://doi.org/10.1101/2022.01.10.475726; this version posted January 12, 2022. The copyright holder for this preprint Fivglawes Bot certified by peer review) is the author/funder, who has granted bioRxiv a license to display the preprint in perpetuity. It is made available under aCC-BY-NC-ND 4.0 International license.

A

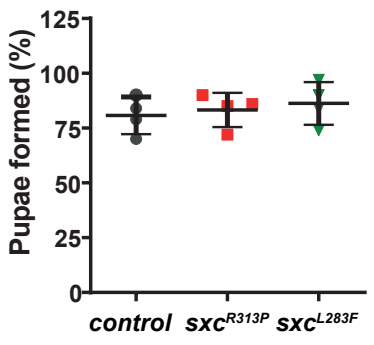

B
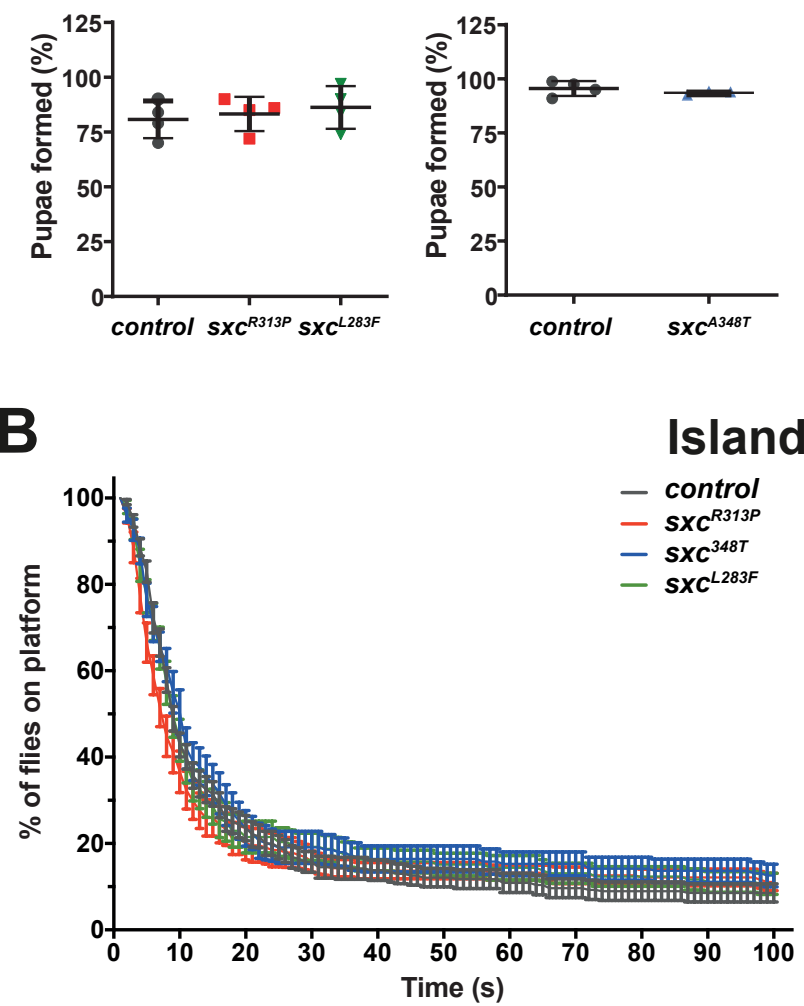

Developmental survival
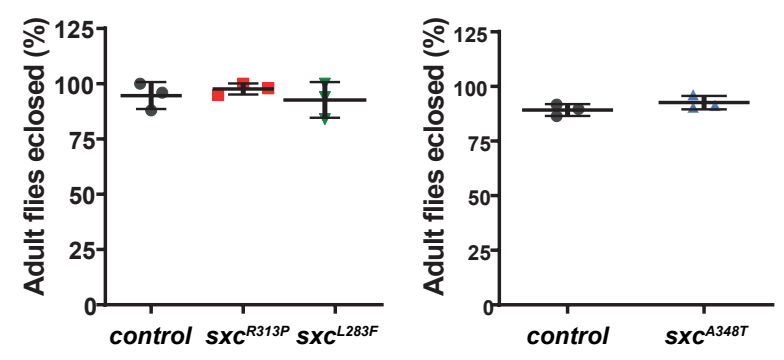

\section{Island assay}

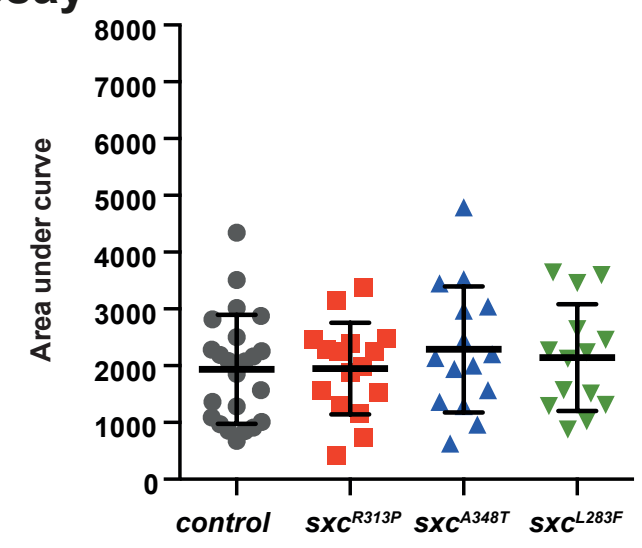

C

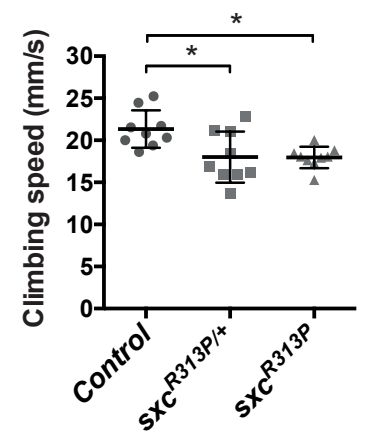

Negative geotaxis assay

D

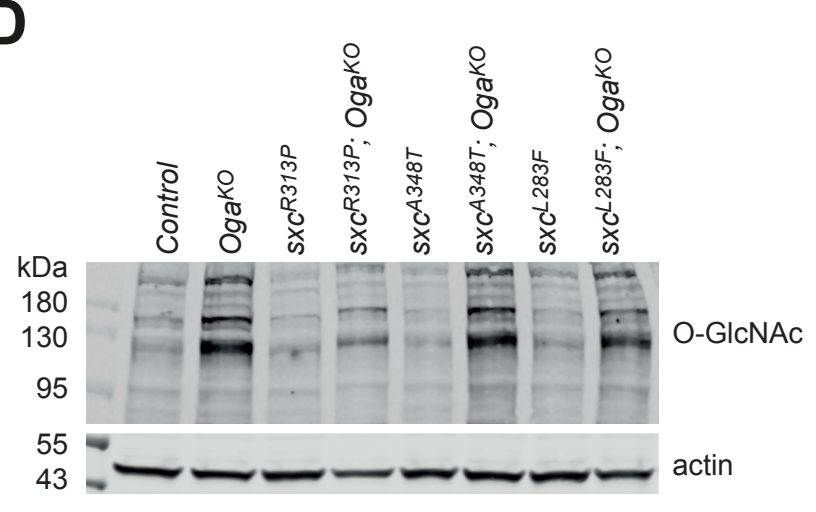

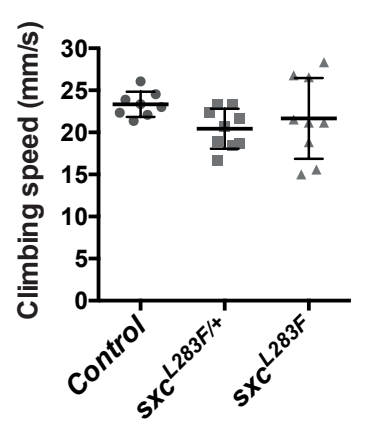

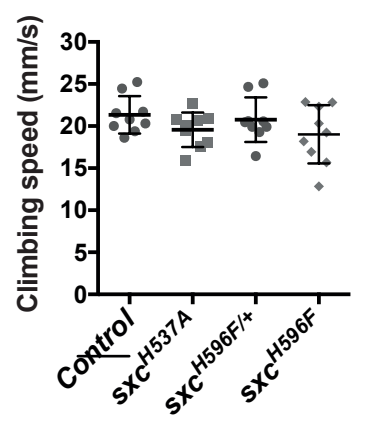

$\mathbf{E}$

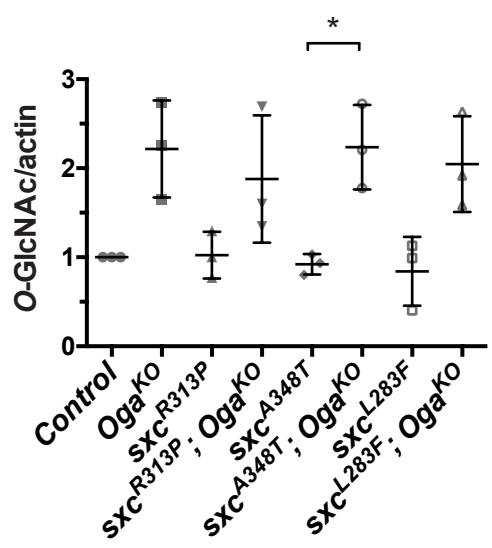


bioRxiv preprint doi: https://doi.org/10.1101/2022.01.10.475726; this version posted January 12, 2022. The copyright holder for this preprint (which was not certified by peer review) is the author/funder, who has granted bioRxiv a license to display the preprint in perpetuity. It is made Figure 4

$\mathbf{A}$

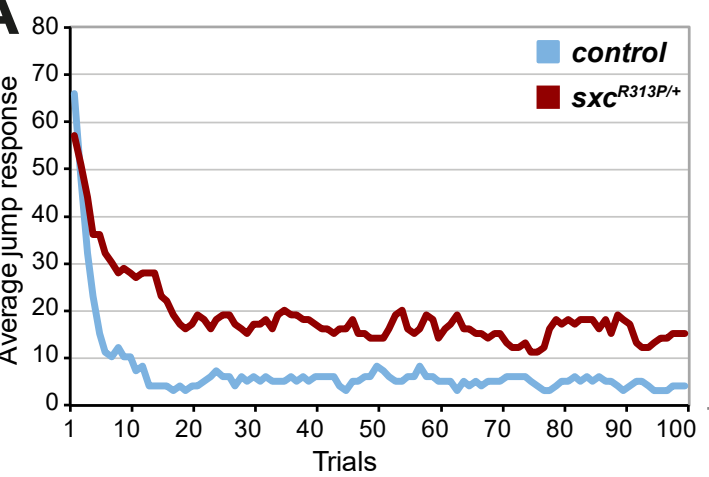

C

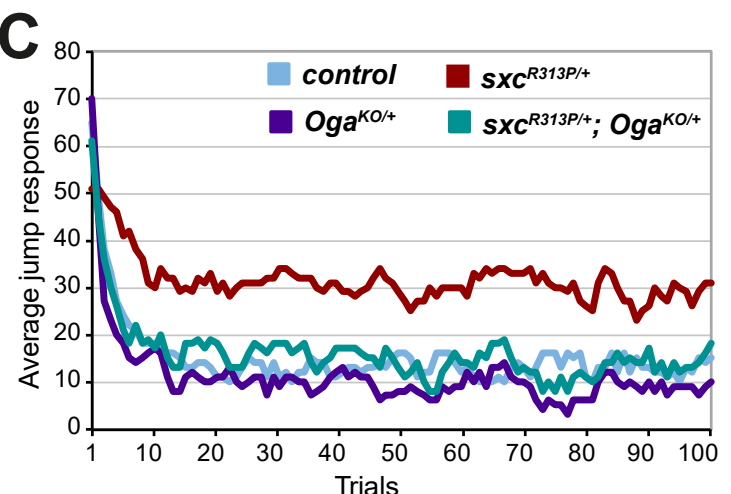

E

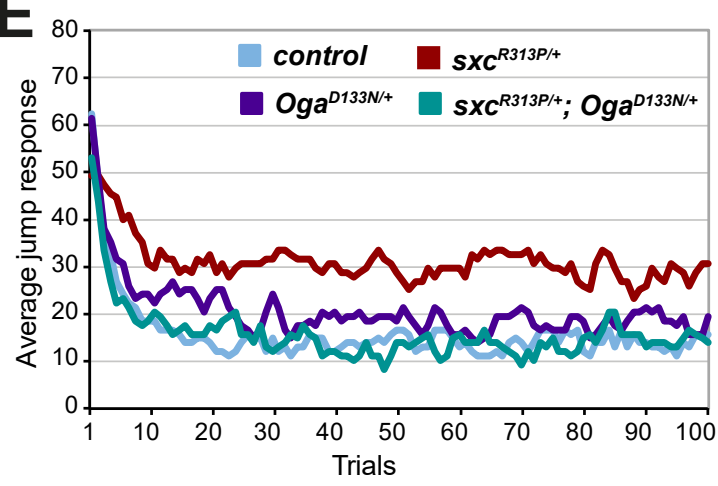

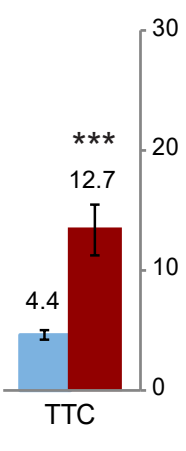

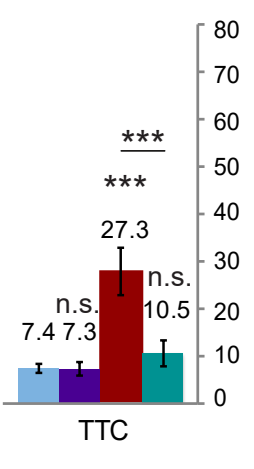

D
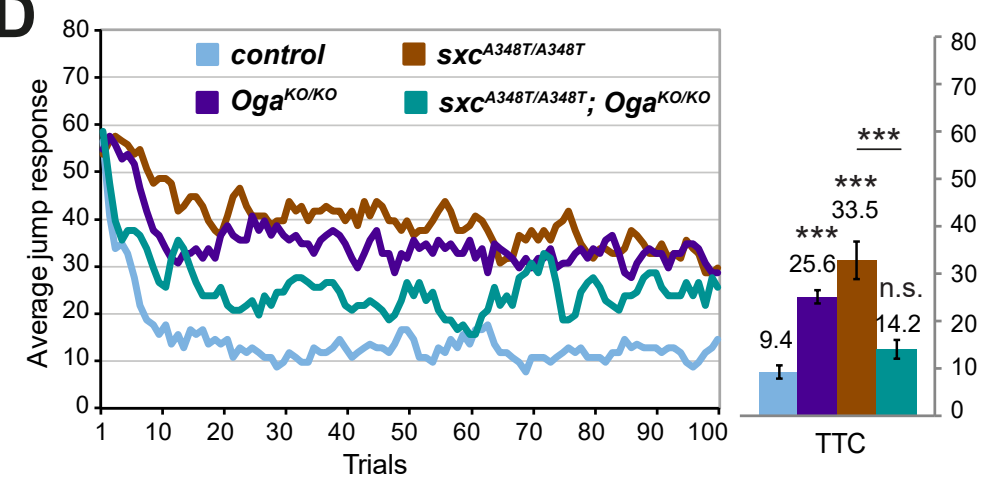

$\mathbf{F}$

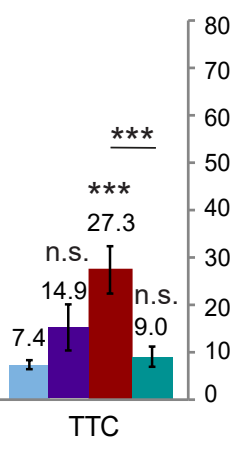

B
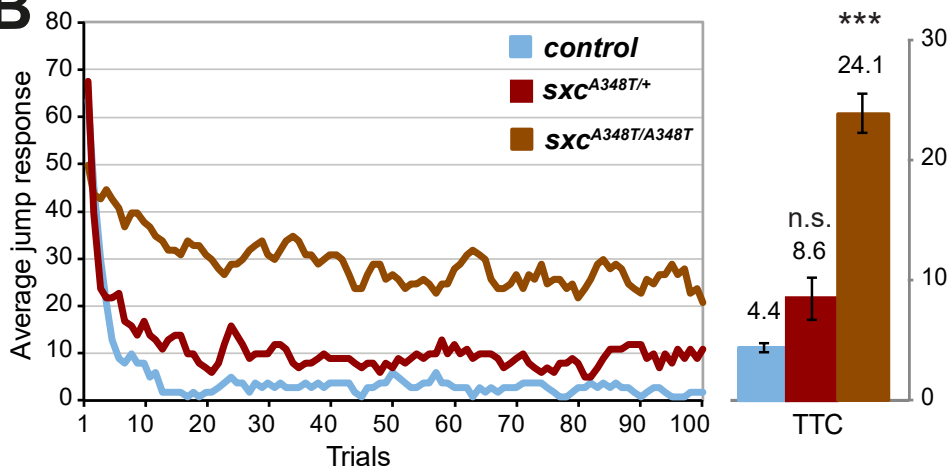

TTC

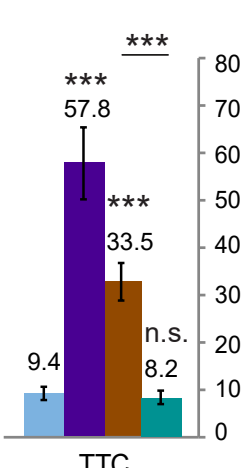


bioRxiv preprint doi: https://doi.org/10.1101/2022.01.10.475726; this version posted January 12, 2022. The copyright holder for this preprint (which was not certified by peer review) is the author/funder, who has granted bioRxiv a license to display the preprint in perpetuity. It is made Figure 5 available under aCC-BY-NC-ND 4.0 International license.

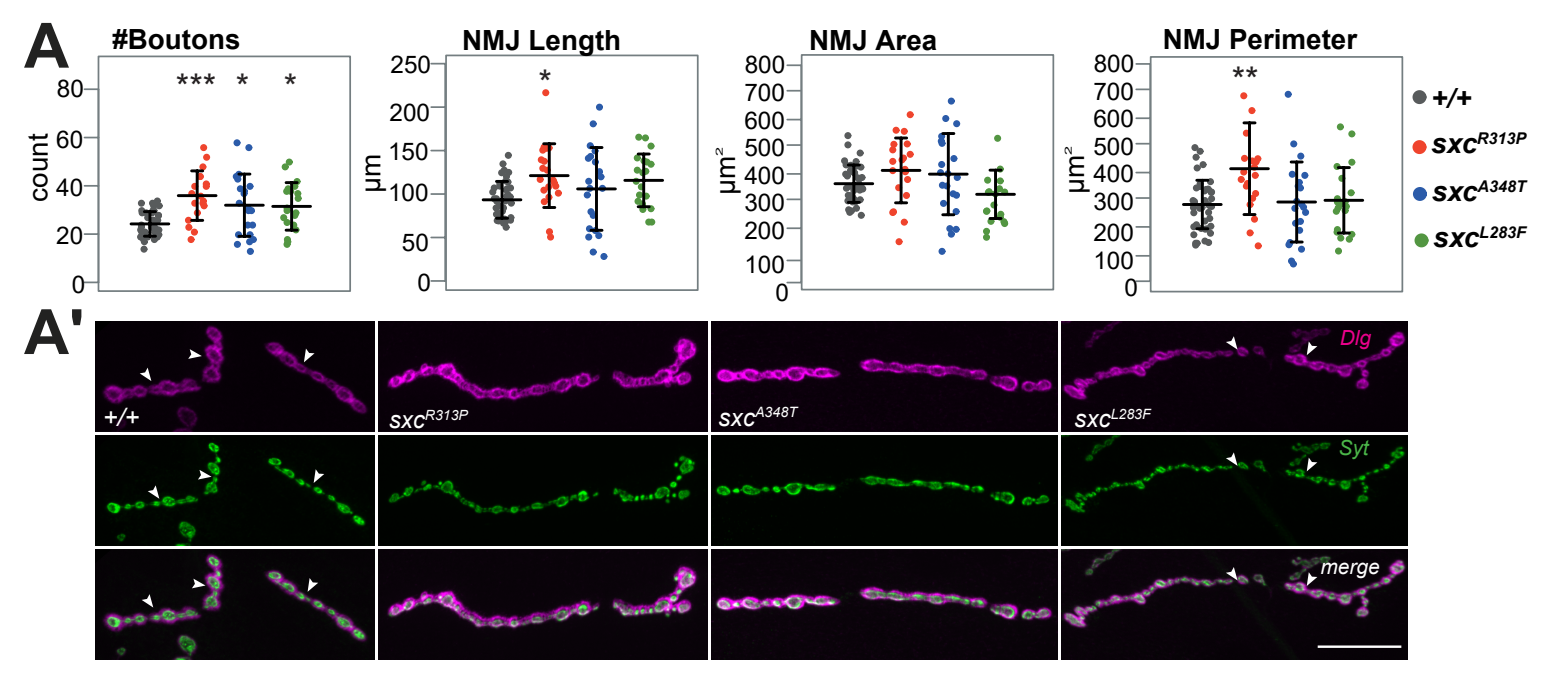

